\author{
SYSTEMS OPTIMIZATION LABORATORY \\ DEPARTMENT OF OPERATIONS RESEARCH \\ STANFORD UNIVERSITY \\ STANFORD, CALIFORNIA 94305-4022
}

\title{
A Strictly Improving Phase I Algorithm Using \\ Least-Squares Subproblems
}

\author{
by
}

S. A. Leichner, G. B. Dantzig and J. W. Davis

TECHNICAL REPORT SOL 92-1

April 1992

\section{DISCLAIMER}

\begin{abstract}
This report was prepared as an account of work sponsored by an agency of the United States Government. Neither the United States Government nor any agency thereof, nor any of their employees, makes any warranty, express or implied, or assumes any legal liability or responsibility for the accuracy, completeness, or usefulness of any information, apparatus, product, or process disclosed, or represents that its use would not infringe privately owned rights. Reference herein to any specific commercial product, process, or service by trade name, trademark, manufacturer, or otherwise does not necessarily constitute or imply its endorsement, recommendation, or favoring by the United States Government or any agency thereof. The views and opinions of authors expressed herein do not necessarily state or reflect those of the United States Government or any agency thereof.
\end{abstract}

Research and reproduction of this report were partially supported by the National Science Foundation Grants ECS-8906260, DMS-8913089; the Department of Energy Grant DE-FG03-92ER25116; Office of Naval Research Grant N00014-89-J-1659; the Electric Power Research Institute Contracts RP 8010-09 at Stanford University, and by Hewlett Packard Laboratories.

Any opinions, findings, and conclusions or recommendations expressed in this publication are those of the authors and do NOT necessarily reflect the views of the above sponsors.

Reproduction in whole or in part is permitted for any purposes of the United States Government. This document has been approved for public release and sale; its distribution is unlimited. 


\title{
A Strictly Improving Phase I Algorithm Using Least-Squares Subproblems
}

\author{
S. A. Leichner \\ G. B. Dantzig \\ J. W. Davis
}

3 April 1992

\begin{abstract}
Although the simplex method's performance in solving linear programming problems is usually quite good, it does not guarantee strict improvement at each iteration on degenerate problems. Instead of trying to recognize and avoid degenerate steps in the simplex method (as some variants do), we have developed a new Phase I algorithm that is completely impervious to degeneracy, with strict improvement attained at each iteration. It is also noted that the new Phase I algorithm is closely related to a number of existing algorithms.

When tested on the 30 smallest $N E T L I B$ linear programming test problerns, the computational results for the new Phase I algorithm were almost 3.5 times faster than the simplex method; on some problems, it was over 10 times faster.
\end{abstract}

\section{Introduction}

On highly degenerate problems, the simplex method often "stalls", performing a number of iterations at a degenerate point before producing any improvement in the objective value. Examples have been constructed by Hoffman [11] and Beale [1] to show that it is theoretically possible that the iterative steps can repeat and thus cycle forever, although this phenomenon is quite rare in practice. Instead of trying to make the simplex method more efficient by trying to avoid stalls due to degeneracy (or near degeneracy), we develop a new Phase I algorithm that is completely impervious to degeneracy.

This new method involves the use of least-squares subproblems in column selection, and is shown to have the property of strict improvement at each iteration, even if every basic solution (in the simplex method sense) is degenerate. Like the simplex method, we will show that the new Phase I algorithm terminates in a finite number of steps, and that in practice, this number is often quite low compared to the simplex method.

Our algorithm is quite similar to a number of existing algorithms, including one to solve the bounded least-squares problem (found in [3]), and another to 
solve the non-negative least-squares problem (found in [12]). In addition, our algorithm is also closely related the algorithm developed by Dantzig [5] and by Van de Panne \& Whinston [14]. R.W. Cottle noted that although this algorithm was designed to solve quadratic programs and thus has a different goal in mind, when applied to an alternate formulation of the Phase I feasibility problem, it is similar to a variant of our least-squares Phase I algorithm.

Section 2 develops most of the theory used by the algorithms presented. It is concerned with finding a feasible solution to a linear program, and after some preliminary results, begins to look at two-variable least-squares problems. The two-variable problem and its positive least-squares solution is used to select the incoming column, and thus build an improved "basic" solution. Conditions are derived under which the solutions to these least-squares problems are strictly positive. Although this is analogous in many ways to the simplex method, it is proved that strict improvement can be guaranteed at each iteration, even in the presence of degeneracy. Next, the detection of infeasibility is discussed, followed by a detailed description of the newly developed least-squares Phase I algorithm, and discussion of a number of variations. Finally, equivalences between our algorithms and the other related algorithms are discussed.

Section 3 presents computational results for the algorithm developed in Section 2. As noted, the least-squares Phase I algorithm has excellent performance, with run times 3.5 times faster than LSSOL's implementation of the simplex method [8]. On some problems, the least-squares Phase I algorithm was over 10 times faster.

Section 4 summarizes the work presented here and attempts to draw some conclusions. Suggestions for future work appear at the end of this section.

\section{Finding a Feasible Solution}

\subsection{The Problem}

The problem to be addressed is that of the Phase I problem solved by the simplex method. That is, find a vector $x$ such that

$$
\begin{aligned}
A x & =b \\
x & \geq 0
\end{aligned}
$$

where $x \in \Re^{n}, b \in \Re^{m}$, and $A \in \Re^{m \times n}$. Denoting the $j$ th column of $A$ by $A_{j}$, we assume $A_{j} \neq 0 \forall j$, and that $b \neq 0$, or else $x=0$ is a trivial solution. Without loss of generality, we also assume that $b$ has been rescaled so that $\|b\|_{2}=1$, and similarly the columns of $A$ have been rescaled so that $\left\|A_{j}\right\|_{2}=1 \forall j$.

Fact 2.1

Let $B$ be an independent subset of the columns of $A$, and let $x_{B}$ satisfy 


$$
\begin{aligned}
B x_{B} & =b \\
x_{B} & >0 .
\end{aligned}
$$

Then by setting $x_{j}=0$ for $A_{j} \notin B$, it is a trivial matter to construct an $x^{*}$ from $x_{B}$ such that $x^{*}$ is a solution to (2-1). (See [6] for a more detailed treatment of the relationship between (2-1) and (2-2).)

The main objective of the least-squares Phase I algorithm to be presented here is to find such a matrix $B$ (if it exists), and positive weightings $x_{B}$, such that $B$ and $x_{B}$ comprise a solution to (2-2). In the remainder of this discussion, we will refer to this matrix $B$ as a basis. Note that this notion of a basis differs from that of the simplex method, as our basis $B$ may contain less than $m$ columns and that these columns may not be sufficient in themselves to span the column space of $A$.

\subsection{Preliminaries}

We need to consider least-squares problems with positive solutions before we can present the algorithm. But before we can consider such problems, we need a few more preliminary facts and results. We will be making use of the Euclidean norm, which we will denote by $\|\cdot\|$.

\section{Fact 2.2}

Let $b, v, p \in \Re^{m}, b \neq 0, p \neq 0, v \neq 0$, and $v \neq b$. Let $u=b-v$. If $1 \cdot v$ is closer to $b$ than any other scalar multiple $\lambda$, then we can conclude that
(a) $v^{T} v=b^{T} v>0$;
(b) $u^{T} v=0$;
(c) $u^{T} u=b^{T} u>0$;
(d) $\quad b^{T} b=u^{T} u+v^{T} v$;
(e) If $p^{\prime r} b \leq 0$, then $1 \cdot v$ is closer to $b$ than any scalar multiple $\mu p$ of $p, \mu \geq 0$;
(f) If $p^{T^{2}} b>0$, then $1 \cdot v$ is closer to $b$ than $\mu p$ for all $\mu \geq 0$ if and only if $\|v\|>p^{T} b /\|p\|$;
(g) If $p \neq 0$ and $v \neq \mu p$, then $v^{T} v p^{T} p-\left(v^{T} p\right)^{2}>0$.

\section{Proof:}

(a) The hypotheses imply that $\lambda=1$ is the solution to

$$
\min _{\lambda}\|b-\lambda v\|^{2} \text {. }
$$


Therefore

$$
\frac{d}{d \lambda}(b-\lambda v)^{T}(b-\lambda v)=0 \text { for } \lambda=1,
$$

and $v^{T} v=b^{T} v$ follows. We also have $b^{T} v>0$ since $v \neq 0$.

(b) Since $u=b-v$ by definition, $u^{T} v=(b-v)^{T} v=0$ by (a).

(c) It follows that $u^{T} u=u^{T}(b-v)=u^{T}$.

(d) We have $b^{T} b=b^{T}(u+v)=u^{T} u+v^{T} v$ by (a) and (c).

(e) The unconstrained minimum of the problem

$$
\min _{\mu}\|b-\mu p\|
$$

occurs at $\mu^{*}=p^{T} b / p^{T} p$, and if $p^{T} b \leq 0$, we have $\mu^{*} \leq 0$. Because $\|b-\mu p\|^{2}$ is a convex function of $\mu$ with a minimum at $\mu^{*}$, we can conclude if $p^{T} b \leq 0$,

$$
\min _{\mu \geq 0}\|b-\mu p\|
$$

must occur at $\mu=0$. Thus,

$$
\min _{\mu \geq 0}\|b-\mu p\|=\|b\| .
$$

Now consider

$$
\|b-v\|=b^{T_{b}}-v^{T} v<\|b\|
$$

If $p^{T} b \leq 0$, we have

$$
\|b-v\|<\|b\|=\min _{\mu \geq 0}\|b-\mu p\| .
$$

(f) From part (e), we know that the minimum of the problem

$$
\min _{\mu}\|b-\mu p\|^{2}
$$

occurs at $\mu^{*}=p^{T} b / p^{T} p$, and we see that if $p^{T} b>0$, we have $\mu^{*}>0$. Thus,

$$
\min _{\mu}\|b-\mu p\|^{2}=\min _{\mu \geq 0}\|b-\mu p\|^{2} .
$$

If $v$ is closer to $b$ than is $\mu p$ for all $\mu \geq 0$, then we have

$$
\begin{aligned}
\|b-v\|^{2} & <\min _{\mu \geq 0}\|b-\mu p\|^{2} \\
b^{T} b-2 v^{T} b+v^{T} v & <\min _{\mu \geq 0}\left(b^{T} b-2 \mu p^{T} b+\mu^{2} p^{T} p\right) \\
-2 v^{T} b+v^{T} v & <-2\left(\frac{p^{T} b}{p^{T} p}\right) p^{T_{b}}+\left(\frac{p^{T} b}{p^{T} p}\right)^{2} p^{T} p \\
-v^{T} v & <-\frac{\left(p^{T} b\right)^{2}}{p^{T} p} \quad \text { by Fact 2.2, part (a). }
\end{aligned}
$$


Therefore, given $p^{T} b>0$, we can say that

$$
\|v\|>\frac{p^{T} b}{\|p\|}
$$

Now if instead of assuming $p^{T} b>0$, we assume that

$$
\|v\|>\frac{p^{T} b}{\|p\|}
$$

we see that the argument reverses exactly. The result follows.

(g) This is a version of the Cauchy-Schwartz inequality.

\section{Fact 2.3}

Given matrix $A \in \Re^{m \times n}(A \neq 0)$, then $A^{T} A$ has full rank if and only if $A$ has full column rank.

\section{Fact 2.4}

Let $\left\{A_{1}, A_{2}, \ldots, A_{k}\right\}$ be a set of linearly independent columns. A unique leastsquares weighting vector $x$ exists, yielding the unconditional minimum of $F=$ $\|b-A x\|$. The unique minimum is found by solving the normal equations $A^{T} A x=A^{T} b$.

\section{Fact 2.5}

Let $\left\{A_{1}, A_{2}, \ldots, A_{k}\right\}$ be a set of linearly independent columns. Let $x^{0}$ be the unique least-squares solution to $\min \left\|b-\sum_{i} A_{i} x_{i}\right\|$, and let $v^{0}=\sum_{i} A_{i} x_{i}^{0}$. Let $x^{1} \neq x^{0}$ be any other weighting of the columns $A_{i}$, and let $v^{1}=\sum_{i} A_{i} x_{i}^{1}$. Then $\left\|b-(1-\lambda) v^{1}-\lambda v^{0}\right\|^{2}$ is monotonically increasing from $\lambda=1$ to $\lambda=0$.

Proof:

$$
\left\|b-(1-\lambda) v^{1}-\lambda v^{0}\right\|^{2}=\left\|b-v^{1}\right\|^{2}-2 \lambda\left(b-v^{1}\right)^{T}\left(v^{0}-v^{1}\right)+\lambda^{2}\left\|v^{1}-v^{0}\right\|^{2}
$$

is a strictly convex function, and its minimum occurs at $\lambda=1$. Thus this quadratic function of $\lambda$ strictly increases from $\lambda=1$ to $\lambda=0$.

\section{Corollary 2.6}

Let $v=(1-\lambda) v^{1}+\lambda v^{0}$. If $v^{0}$ is closer $t c b$ than is $v^{1}$, then $u^{T} u<u_{1}^{T} u_{1}$, where $u_{1}=b-v^{1}$, and $u=b-v$ for all $0<\lambda<1$.

Proof: Let $u_{0}=b-v^{0}$. We know that $u_{0} \neq u_{1}$ because $u_{0}^{T} u_{0}<u_{1}^{T} u_{1}$. Therefore

$$
\left\|u_{1}-u_{0}\right\|^{2}=u_{1}^{T} u_{1}+u_{0}^{T} u_{0}-2 u_{1}^{T} u_{0}>0
$$


and $2 u_{1}^{T} u_{0}<u_{1}^{T} u_{1}+u_{0}^{T} u_{0}$. Hence,

$$
\begin{aligned}
u^{T} u & =\left(\lambda u_{0}+(1-\lambda) u_{1}\right)^{T}\left(\lambda u_{0}+(1-\lambda) u_{1}\right) \\
& =\lambda^{2} u_{0}^{T} u_{0}+(1-\lambda)^{2} u_{1}^{T} u_{1}+2 \lambda(1-\lambda) u_{1}^{T} u_{0} \\
& <\lambda^{2} u_{0}^{T} u_{0}+(1-\lambda)^{2} u_{1}^{T} u_{1}+\lambda(1-\lambda)\left(u_{1}^{T} u_{1}+u_{0}^{T} u_{0}\right) \\
& =(1-\lambda) u_{1}^{T} u_{1}+\lambda u_{0}^{T} u_{0} \\
& \left.<(1-\lambda) u_{1}^{T} u_{1}+\lambda u_{1}^{T} u_{1} \quad \text { (because } u_{0}^{T} u_{0}<u_{1}^{T} u_{1}\right) \\
& =u_{1}^{T} u_{1} .
\end{aligned}
$$

Thus any nontrivial convex combination of two vectors $v^{0}$ and $v^{1}$ as shown above is strictly closer to $b$ than whichever vector $v^{0}$ or $v^{1}$ is farthest away from $b$.

\section{Corollary 2.7}

Let $A \in \Re^{m \times n}$ be made up of linearly independent columns, and let $x^{0}$ be the unique least-squares solution to $\min \|b-A x\|$. Let $v^{0}=A x^{0}$. Assume $v^{0} \geq 0$. If $v^{1}>0$, where $v^{1}$ is otherwise arbitrary, then a convex combination $v$ of $v^{0}$ and $v^{1}$ can be found to generate $a v \geq 0$ closer to $b$ than $v^{1}$ for some $\lambda, 0 \leq \lambda<1$.

Proof: From Fact 2.5, we know that $\left\|b-(1-\lambda) v^{1}-\lambda v^{0}\right\|^{2}$ is monotonically increasing from $\lambda=1$ to $\lambda=0$. Thus any convex combination, $v=(1-\lambda) v^{1}+$ $\lambda v^{0}$, has the property that

$$
\left\|b-v^{1}\right\|^{2}>\|b-v\|^{2}>\left\|b-v^{0}\right\|^{2} \quad(\lambda \neq 0, \lambda \neq 1)
$$

If we want $v \geq 0$ with $v$ closer to $b$ than $v^{1}$, start with $\lambda=1$ and decrease $\lambda$ until all components of $v$ are $\geq 0$. This is possible because $v^{1}>0$.

\section{Fact 2.8}

Given $x>0, y \geq 0$, the minimum $\lambda$ required to make the vector $\lambda x+(1-\lambda) y \geq 0$ is

$$
\lambda^{*}=\max _{y_{i}<0} \frac{-y_{i}}{x_{i}-y_{i}} .
$$

This concludes the necessary preliminary results.

\subsection{Least Squares with Positive Solutions}

In this section, we will consider the conditions under which the solution to the (unconstrained) two-variable least-squares problem

$$
\min F(\alpha, \beta)=\min _{\alpha, \beta}\|b-\alpha v-\beta p\|^{2}
$$

is unique, and the implications of a non-positive solution. Then we will derive conditions under which the solution is strictly positive. 


\section{Theorem 2.9}

Let $p \neq 0, v \neq \mu p$ and $u=b-v$. Let $\min F=F_{0}$. Then,

(a) the values of $\left(\alpha_{0}, \beta_{0}\right)$ yielding $F_{0}$ are unique;

(b) $F_{0}=u^{T} u$ if and only if $\left(\alpha_{0}, \beta_{0}\right)=(1,0)$.

\section{Proof:}

(a) Noting that the condition $v \neq \mu p$ implies $v$ and $p$ are linearly independent, this follows directly from Fact 2.4 .

(b) If $\left(\alpha_{0}, \beta_{0}\right)=(1,0)$, then we see that $F_{0}=u^{T} u$. By part (a), we know that the values of $\left(\alpha_{0}, \beta_{0}\right)$ yielding $F_{0}$ are unique. Therefore, the converse also holds; that is, $F_{0}=u^{T} u=\|b-1 \cdot v-0 \cdot p\|^{2}$ implies $\left(\alpha_{0}, \beta_{0}\right)=(1,0)$.

\section{Corollary 2.10}

Let $1 \cdot v$ be closer to $b$ than any other $\lambda v$. If $F_{0} \neq u^{T} u$, then $F_{0}<u^{T} u$ and $\beta_{0} \neq 0$. In addition, if $\min \left\{\alpha_{0}, \beta_{0}\right\}<0$, then $F_{0}<u^{T} u$.

Proof: If $F_{0} \neq u^{T} u$, we can conclude that $F_{0}<u^{T} u$, as $F=u^{T} u$ is obtainable at $(\alpha, \beta)=(1,0)$.

Now assume that $F_{0}<u^{T} u$ and $\beta_{0}=0$. Thus, $\alpha_{0} v+\beta_{0} p=\alpha_{0} v$. Since $v$ is closer to $b$ than is any $\lambda v(\lambda \neq 1), \alpha_{0} v$ cannot be as close to $b$ as is $v$ for $\alpha_{0} \neq 1$. Therefore if $\beta_{0}=0$, then $\alpha_{0}=1$. From Theorem 2.9, we know that if $\left(\alpha_{0}, \beta_{0}\right)=(1,0)$, then $F=u^{T} u$, which is a contradiction.

In Theorem 2.9, we showed that $F_{0}=u^{T} u$ if and only if $\left(\alpha_{0}, \beta_{0}\right)=(1,0)$. We just showed that if $F_{0} \neq u^{T} u$, then $F_{0}<u^{T} u$. If $\min \left\{\alpha_{0}, \beta_{0}\right\}<0$, then $\left(\alpha_{0}, \beta_{0}\right) \neq(1,0)$. Thus we can conclude that $F_{0} \neq u^{T} u$, which in turn implies that $F_{0}<u^{T} u$.

\section{Theorem 2.11}

Let $1 \cdot v$ be closer to $b$ than any other $\lambda v$. Also let $v \neq \mu p$ and let $v$ be closer to $b$ than any $\mu p, \mu \geq 0$. Let $F_{0}=\min F$, and let $\left(\alpha_{0}, \beta_{0}\right)$ be the unique solution corresponding to $F_{0}$. Let $v_{0}=\alpha_{0} v+\beta_{0} p$. If $\min \left\{\alpha_{0}, \beta_{0}\right\}<0$, then there exist no $\left(\alpha_{1}, \beta_{1}\right) \geq 0$ such that $v_{1}=\alpha_{1} v+\beta_{1} p$ is closer to $b$ than is $v$.

Proof: Assume $\exists\left(\alpha_{1}, \beta_{1}\right) \geq 0$ such that $v_{1}$ is closer to $b$ than is $v$, i.e., $\|b-v\|>$ $\left\|b-v_{1}\right\|$.

Case 1: $\alpha_{0}<0, \beta_{0} \geq 0$.

Let $\tilde{v}$ be a convex combination of $v_{0}$ and $v_{1}$ :

$$
\begin{aligned}
\tilde{v} & =\lambda v_{0}+(1-\lambda) v_{1} \\
& =\left(\lambda \alpha_{0}+(1-\lambda) \alpha_{1}\right) v+\left(\lambda \beta_{0}+(1-\lambda) \beta_{1}\right) p \\
& =\tilde{\alpha} v+\tilde{\beta} p
\end{aligned}
$$


where $\tilde{\alpha}=\lambda \alpha_{0}+(1-\lambda) \alpha_{1}$ and $\tilde{\beta}=\lambda \beta_{0}+(1-\lambda) \beta_{1}$.

Since $v_{0}$ is formed from the unique solution to $\min F$, we know that $v_{0}$ is closer to $b$ than is $v_{1}$. Thus by Corollary 2.6, we can say that any convex combination of $v_{0}$ and $v_{1}$ is at least as close to $b$ as is $v_{1}$. That is, $\|b-\tilde{v}\| \leq$ $\left\|b-v_{1}\right\|$. At some point between $v_{0}$ and $v_{1}, \tilde{\alpha}=0$, since $\alpha_{0}<0$ and $\alpha_{1} \geq 0$. At such a point, we have $\tilde{v}=\tilde{\beta} p$. However, we know that $v$ is closer to $b$ than any $\beta p$ for $\beta \geq 0$, so $v$ is closer to $b$ than is $\tilde{v}$. That is, $\|b-v\|<\|b-\tilde{v}\|$. We also know that $\tilde{v}$ is at least as close to $b$ as is $v_{1}$, i.e., $\|b-\tilde{v}\| \leq\left\|b-v_{1}\right\|$. Thus we have $\|b-v\|<\left\|b-v_{1}\right\|$, which contradicts the assumption.

Case 2: $\beta_{0}<0$, no conditions on $\alpha_{0}$.

As in Case 1, let $\tilde{v}$ be a convex combination of $v_{0}$ and $v_{1}$ :

$$
\begin{aligned}
\tilde{\boldsymbol{v}} & =\lambda v_{0}+(1-\lambda) v_{1} \\
& =\tilde{\alpha} v+\tilde{\beta} p,
\end{aligned}
$$

where $\tilde{\alpha}$ and $\tilde{\beta}$ are as defined above in Case 1 . Also as in Case 1 , we can conclude that any convex combination of $v_{0}$ and $v_{1}$ is at least as close to $b$ as is $v_{1}$. That is, $\|b-\tilde{v}\| \leq\left\|b-v_{1}\right\|$. At some point between $v_{0}$ and $v_{1}$, we have $\tilde{\beta}=0$. At such a point, $\tilde{v}=\tilde{\alpha} v$. However, we know that $v$ is closer to $b$ than any $\alpha v$ for $\alpha \neq 1$. So $v$ is at least as close to $b$ as is $\tilde{v}$, i.e., $\|b-v\| \leq\|b-\tilde{v}\|$. We also know that $\|b-\tilde{v}\| \leq\left\|b-v_{1}\right\|$. Thus we have $\|b-v\| \leq\left\|b-v_{1}\right\|$, which again contradicts the assumption.

Now we will derive conditions under which the solution $\left(\alpha_{0}, \beta_{0}\right)$ to

$$
\min F=\min _{\alpha, \beta}\|b-\alpha v-\beta p\|^{2}
$$

is strictly positive.

\section{Theorem 2.12}

Let $v \neq \mu p, p \neq 0$. Let $v$ be closer to $b$ than any $\lambda v, \lambda \neq 1$ and let $u=b-v$. Let $F$ and $\left(\alpha_{0}, \beta_{0}\right)$ be as defined in Theorem 2.11. Then $\beta_{0}>0$ if and only if $p^{T} u>0$.

Proof: Solving for $\beta_{0}$ at the minimum of $F$, we get

$$
\beta_{0}=\frac{b^{T} p v^{T} v-b^{T} v p^{T} v}{p^{T} p v^{T} v-\left(p^{T} v\right)^{2}}
$$

Consider the sign of $\beta_{0}$. From Fact 2.2, we know that $p^{T} p v^{T} v-\left(p^{T} v\right)^{2}>0$, so we need only consider the numerator:

$$
\begin{aligned}
p^{T} b v^{T} v-b^{T} v p^{T} v & =p^{T} b v^{T} v-p^{T} v v^{T} v \quad \text { (by Fact 2.2, part (a)) } \\
& =v^{T} v p^{T} u .
\end{aligned}
$$

Since $v \neq 0$, we know that $v^{T} v>0$. Thus the sign of $p^{T} u$ determines the sign of the numerator and hence the sign of $\beta_{0}$. The result follows. 


\section{Theorem 2.13}

Given the same conditions as in Theorem 2.12 with the addition that $v$ be closer to $b$ than is $\mu p$ for $\mu \geq 0$, then $\alpha_{0}>0$ if $p^{T} u>0$.

Proof: Solving for $\alpha_{0}$ at the minimum of F, we get

$$
\alpha_{0}=\frac{v^{T} v p^{T} p-\left(p^{T} v\right)^{2}-p^{T} u p^{T} v}{p^{T} p v^{T_{v}}-\left(p^{T} v\right)^{2}} .
$$

From Fact 2.2, we know that $p^{T} p v^{T} v-\left(p^{T} v\right)^{2}>0$, so the sign of $\alpha_{0}$ is the same as the sign of the numerator $v^{T} v p^{T} p-\left(p^{T} v\right)^{2}-p^{T} u p^{T} v$.

Case 1: $p^{T} b>0$ :

From Fact 2.2 we know that $v^{T} v p^{T} p>\left(p^{T} b\right)^{2}$. Therefore,

$$
\begin{aligned}
v^{T} v p^{T} p-\left(p^{T} v\right)^{2}-p^{T} u p^{T} v & >\left(p^{T} b\right)^{2}-\left(p^{T} v\right)^{2}-p^{T} v p^{T} u \\
& =\left(p^{T} b\right)^{2}-\left(p^{T} v\right)^{2}-p^{T} v p^{T} b+\left(p^{T} v\right)^{2} \\
& =p^{T} b\left(p^{T} b-p^{T} v\right) \\
& =p^{T} b p^{T} u>0
\end{aligned}
$$

since $p^{T_{b}}>0$ and $p^{T} u>0$. Thus in Case $1, \alpha_{0}>0$ if $p^{T} u>0$.

Case 2: $p^{T} b \leq 0$ :

We know $\bar{p}^{T} u>0$ and thus $p^{T} b-p^{T} v>0$. But $p^{T} b \leq 0$, so $p^{T} v$ must be negative. Again we consider the sign of the numerator of $\alpha_{0}$. By Fact 2.2, we know $v^{T} v p^{T} p-\left(p^{T} v\right)^{2}>0$, so consider $-p^{T} u p^{T} v$. However, we know that $p^{T} u>0$ and $p^{T} v<0$. Thus $-p^{T} u p^{T} v>0$. Thus in Case $2, \alpha_{0}>0$ if $p^{T} u>0$.

\section{Corollary 2.14}

Given the conditions of Theorem 2.13, the unconditional minimum of $F$ is at $\left(\alpha_{0}, \beta_{0}\right)>0$ if $u^{T} p>0$.

Proof: This follows directly from Theorems 2.12 and 2.13.

\subsection{Column Selection Criteria}

We have considered the problem (2-3), and conditions under which $\left(\alpha_{0}, \beta_{0}\right)>0$. So far, we have held both $v$ and $p$ fixed. We now consider holding only $v$ fixed, but allowing $p$ to be chosen as one of the columns of the matrix $A$ from (2-1). In particular, we select that column $A_{s}$ whose nonnegative combination with $v$ brings us closer to $b$ than any other $A_{j}, j \neq s$. This is done by solving the problem

$$
\min G=\min _{j}\left(\min _{\alpha, \beta}\left\|b-\alpha v-\beta A_{j}\right\|^{2}\right)
$$


Before finding the minimum of $G$ in the general case, we will first consider the simpler problem of finding $A_{j}=A_{s}$ that yields the minimum of $G$ when $v=0$. The solution $\beta=\beta_{0}$ will be used to set $v=\beta_{0} A_{s}$ as the initial approximation to $b$.

Theorem 2.15

If $b^{T} A_{j}>0$ for some $j$, then the solution to

$$
\min _{j}\left(\inf _{\beta}\left\|b-\beta A_{j}\right\|^{2}\right)
$$

is attained at $\beta_{0}=b^{T} A_{s}$ where

$$
s=\underset{j}{\operatorname{argmax}} b^{T} A_{j} .
$$

Otherwise, if $b^{T} A_{j} \leq 0$ for all $j$, then no matter which $j$ is chosen, the inf of (25) is attained at $\beta=0$. In the latter case, the closest nonnegative approximation to $b$ is given by $x=0$.

Proof: First consider

$$
\min F=\min _{\beta}\left\|b-\beta A_{j}\right\|^{2} .
$$

Because the columns of $A$ are normalized, the minimum of $F$ occurs at

$$
\beta_{0}=\frac{b^{T} A_{j}}{A_{j}^{T} A_{j}}=b^{T} A_{j}
$$

Evaluating $F$ at $\beta_{0}$, we get

$$
\begin{aligned}
\left.F\right|_{\beta=\beta_{0}} & =\left(b-\beta_{0} A_{j}\right)^{T}\left(b-\beta_{0} A_{j}\right) \\
& =b^{T} b-\left(b^{T} A_{j}\right)^{2} .
\end{aligned}
$$

In choosing a particular $A_{j}$, we want to minimize this value subject to $\beta_{0}>0$. This corresponds to maximizing $\left(b^{T} A_{j}\right)^{2}$ over all positive $b^{T} A_{j}$. If $b^{T} A_{j} \leq 0$ for all $j$, then the inf of problem (2-5) is clearly attained at $\beta=0$, regardless of the choice of $j$.

We set $v=b^{T} A_{s} A_{s}$ as the initial approximation to $b$ (where $s$ is defined by $(2-6)$ ), and we proceed to seek to improve this approximation by considering the problem (2-4). For convenience of discussion, we assume that $s$ defined by $(2-6)$ is unique, although this is not a necessary condition. 


\section{Theorem 2.16}

Let $1 \cdot v$ be closer to $b$ than any scalar multiple $\lambda v$ for $\lambda \neq 1$. Let $\mu A_{j} \neq v \forall j$, where the columns $A_{j}$ are normalized as in (2-1). Let $v$ be closer to $b$ than $\mu A_{j}$ for $\mu \geq 0$ and all $j$, and let $u=b-v$. Under these conditions, the solution to

$$
\min _{j}\left(\inf _{(\alpha, \beta)>0}\left\|b-\alpha v-\beta A_{j}\right\|^{2}\right)
$$

is attained at $j=s$ where

$$
s=\underset{j}{\operatorname{argmax}} \frac{A_{j}^{T} u}{\left(v^{T} v-\left(A_{j}^{T} v\right)^{2}\right)^{1 / 2}},
$$

provided that $\exists j: A_{j}^{T} u>0$. If $\exists$ no $j$ such that $A_{j}^{T} u>0$, then any $(\alpha, \beta) \geq 0$ will produce a value of (2-7) that is greater than or equal to $\|b-v\|^{2}$.

Proof: First consider

$$
\min F_{j}=\min _{\alpha, \beta}\left\|b-\alpha v-\beta A_{j}\right\|^{2} .
$$

From the proofs of Theorem 2.12 and Theorem 2.13, we know that the unconditional minimum of $F_{j}$ occurs at $\left(\alpha_{j}, \beta_{j}\right)$ where

$$
\left(\alpha_{j}, \beta_{j}\right)=\left(\frac{A_{j}^{T} A_{j} v^{T} v-A_{j}^{T} v A_{j}^{T} b}{A_{j}^{T} A_{j} v^{T} v-\left(A_{j}^{T} v\right)^{2}}, \frac{v^{T} v A_{j}^{T} u}{A_{j}^{T} A_{j} v^{T} v-\left(A_{j}^{T} v\right)^{2}}\right) .
$$

Since $A_{j}^{T} A_{j}=1$, this can be rewritten as

$$
\left(\alpha_{j}, \beta_{i}\right)=\left(\frac{v^{T} v-A_{j}^{T} v A_{j}^{T} b}{v^{T} v-\left(A_{j}^{T} v\right)^{2}}, \frac{v^{T} v A_{j}^{T} u}{v^{T} v-\left(A_{j}^{T} v\right)^{2}}\right) .
$$

Evaluating $F_{j}$ at the minimum $\left(\alpha_{j}, \beta_{j}\right)$, we get

$$
\begin{aligned}
\left.F_{j}\right|_{\substack{\alpha=\alpha_{j} \\
\beta=\beta_{j}}} & =\left(b-\alpha_{j} v-\beta_{j} A_{j}\right)^{T}\left(b-\alpha_{j} v-\beta_{j} A_{j}\right) \\
& =b^{T}\left(b-\alpha_{j} v-\beta_{j} A_{j}\right)-\alpha_{j} v^{T}\left(b-\alpha_{j} v-\beta_{j} A_{j}\right)-\beta_{j} A_{j}^{T}\left(b-\alpha_{j} v-\beta_{j} A_{j}\right) \\
& =b^{T}\left(b-\alpha_{j} v-\beta_{j} A_{j}\right) \\
& =b^{T} b-\alpha_{j} v^{T} v-\beta_{j} b^{T} A_{j} \quad(\text { from Fact } 2.2, \text { part (a)) } \\
& =b^{T} b-\left(\frac{\left(v^{T} v\right)^{2}-v^{T} v b^{T} A_{j} v^{T} A_{j}}{v^{T} v-\left(v^{T} A_{j}\right)^{2}}\right)-\left(\frac{v^{T} v A_{j}^{T} u b^{T} A_{j}}{v^{T} v-\left(v^{T} A_{j}\right)^{2}}\right) \\
& =b^{T} b-v^{T} v\left(\frac{v^{T} v-2 b^{T} A_{j} v^{T} A_{j}+\left(A_{j}^{T} b\right)^{2}}{v^{T} v-\left(v^{T} A_{j}\right)^{2}}\right) .
\end{aligned}
$$


We choose $A_{s}$ so as to minimize $F_{j}$. This corresponds to maximizing the following over $A_{j}$ :

$$
\left(\frac{v^{T} v-2 b^{T} A_{j} v^{T} A_{j}+\left(b^{T} A_{j}\right)^{2}}{v^{T} v-\left(v^{T} A_{j}\right)^{2}}\right)=\frac{\left(A_{j}^{T}(b-v)\right)^{2}}{v^{T} v-\left(A_{j}^{T} v\right)^{2}}+1,
$$

which is the same as choosing $j=s$ so as to maximize

$$
\frac{\left(A_{j}^{T}(b-v)\right)^{2}}{v^{T} v-\left(A_{j}^{T} v\right)^{2}}=\frac{\left(A_{j}^{T} u\right)^{2}}{v^{T} v-\left(A_{j}^{T} v\right)^{2}} .
$$

Let us consider tho solution $\left(\alpha_{0}, \beta_{0}\right)$ to (2-9). Let $\min F=F\left(\alpha_{0}, \beta_{0}\right)=F_{0}$. Note that by Theorem 2.9 , the solution $\left(\alpha_{0}, \beta_{0}\right)$ is unique, and any other solution $\left(\alpha_{1}, \beta_{1}\right) \neq\left(\alpha_{0}, \beta_{0}\right)$ must have the property that $F\left(\alpha_{1}, \beta_{1}\right)>F\left(\alpha_{0}, \beta_{0}\right)$.

Case 1: $u^{r} A_{j}=0$

From the proof of Theorem 2.12 and Theorem 2.13, we know that $\left(\alpha_{0}, \beta_{0}\right)=$ $(1,0)$, and $F\left(\alpha_{0}, \beta_{0}\right)=\|b-v\|^{2}$. If we were to insist that we minimize over strictly positive $(\alpha, \beta)$, we would get an $\left(\alpha_{1}, \beta_{1}\right) \geq\left(\epsilon_{1}, \epsilon_{2}\right)>0$ for some $\left(\epsilon_{1}, \epsilon_{2}\right)$, and $\left(\alpha_{1}, \beta_{1}\right) \neq\left(\alpha_{0}, \beta_{0}\right)$. Thus from Theorem 2.9 and Corollary 2.10, $F\left(\alpha_{1}, \beta_{1}\right)>$ $F\left(\alpha_{0}, \beta_{0}\right)$, or in other words, $F\left(\alpha_{1}, \beta_{1}\right)>\|b-v\|^{2}$.

Case 2: $u^{T} A_{j}<0$

From the proof of Theorem 2.12, we see that $\beta_{0}<0$. From Theorem 2.11, we know that there is no $\left(\alpha_{1}, \beta_{1}\right) \geq 0$ such that $F\left(\alpha_{1}, \beta_{1}\right)<\|b-v\|^{2}$. Therefore if we were to insist that we minimize over strictly positive $(\alpha, \beta)$, we would get an $\left(\alpha_{1}, \beta_{1}\right) \geq\left(\epsilon_{1}, \epsilon_{2}\right)>0$ such that $F\left(\alpha_{1}, \beta_{1}\right) \geq\|b-v\|^{2}$.

Case 3: $u^{T} A_{j}>0$

From Corollary 2.14, we know that $\left(\alpha_{0}, \beta_{0}\right)>0$. We also know that $F=$ $\|b-v\|^{2}$ is attainable with $(\alpha, \beta)=(1,0)$. Because the solution is unique, we can conclude that $F(1,0)>F\left(\alpha_{0}, \beta_{0}\right)$, or in other words, $F\left(\alpha_{0}, \beta_{0}\right)<\|b-v\|^{2}$.

Only in Case 3 are we able to find an $(\alpha, \beta)>0$ such that

$$
\min _{(\alpha, \beta)>0}\left\|b-\alpha v-\beta A_{j}\right\|^{2}<\|b-v\|^{2} .
$$

Therefore, the only columns to consider to improve the approximation $v$ are those such that $u^{T} A_{j}>0$, provided that any such columns exist. Thus from the solution we computed for problem (2-9), we see that the solution to to (2-7) is given by

$$
s=\underset{j \cdot A_{j}^{T_{u}>0}}{\operatorname{argmax}} \frac{A_{j}^{T} u}{\left(v^{T} v-\left(A_{j}^{T} v\right)^{2}\right)^{1 / 2}},
$$

provided that $\exists j: A_{j}^{T} u>0$. We also showed that if $A^{T} u \leq 0$, then any solution with $(\alpha, \beta) \geq 0$ will produce a minimum of $(2-7)$ that is $\geq\|b-v\|^{2}$ (and hence $(\alpha, \beta)=(1,0)$ is as good a solution as any possible $)$. 
We will eventually consider more carefully the implications of the situation when $A^{T} u \leq 0$, but we need more results first.

\subsection{Basic Weightings}

A set of linearly independent columns of $A,\left\{A_{1}, A_{2}, \ldots, A_{k}\right\}$, will be denoted by $B$, and be called a basis; the set of indices $\{1,2, \ldots, k\}$ will be referred to as basic indices. A nonnegative weighting of a basis is any' $v=B x_{B}$ with $x_{B} \geq 0$. It is a positive weighting if $x_{B}>0$. A positive weighting such that $v$ is closer to $b$ than is any other positive weighting will be referred to as a basic weighting.

Given a basis, there may or may not exist a positive weighting that is closest to $b$. That is to say, the closest nonnegative weighting may have some weights $\left(x_{B}\right)_{i}=0$, in which case it is a basic weighting for the subset of columns of $B$ where $\left(x_{B}\right)_{i}>0$.

Now consider the least-squares problem

$$
\min \left\|b-B x_{B}\right\|^{2}
$$

Let $x_{B}^{0}$ be the solution and let $v^{0}=B x_{B}^{0}$. We will refer io such a $v^{0}$ as the least-squares weighting of $B$. Notice that this implies that a nositive weighting of a basis $B$ is a basic weighting if and only if it is the least-squares weighting of the columns of $B$.

Fact 2.17

Any bosic weighting $v$ of a basis $B$ is unique.

\section{Theorem 2.18}

Let $v=B x_{B}$ be a positive weighting of the columns of $B$. Let $u=b-v$. Then a necessary and sufficient condition that $v$ be a basic weighting is $u^{T} B=0$.

Proof: We know that if $v$ is a positive weighting, then $v$ is a basic weighting if and only if it is a least-squares weighting. Thus it is equivalent to prove that a necessary and sufficient condition that $v$ be a leasi-squares weighting is $u^{T} B=0$. The normal equations, which are necessary and sufficient conditions for $v$ to be the least-squares weighting, are

$$
B^{T}\left(B x_{B}-b\right)=0
$$

Noting $B x_{B}-b=v-b=u$, the result follows.

\section{Corollary 2.19}

Given $v$, a basic weighting corresponding $t_{0} B$, and $u^{T} A_{s} \neq 0$ where $u=b-v$, then $\left\{B, A_{s}\right\}$ is a set of linearly independent columns.

Proof: By Theorem 2.18, $u^{T} B=0$ because $v$ is a bissic weighting. Since $u^{T} A_{s} \neq 0$, we know that $A_{s}$ is not containec' in the space spanned by the columns of $B$. (Otherwise $A_{8}$ would be a linear combination of the columns of $B$, and we would have $u^{T} A_{s}=0$.) 


\section{Corollary 2.20}

If $v$ is a basic weighting corresponding to $B$, and $u=b-v$, then if we select $A_{s}$ by the criterion (2-8), where $A_{s}^{T} u>0$, then $A_{s}$ is linearly independent of the columns in $B$.

Proof: This follows directly from Corollary 2.19 , as $A_{s}^{T} u \neq 0$.

Corollary 2.21

If $A_{s}$ is chosen as in Corollary 2.20, then the least-squares problem

$$
\min \left\|b-B x_{B}-A_{s} x_{s}\right\|^{2}
$$

has a unique solution.

Proof: $B$ has linearly independent columns. By Corollary $2.20,\left\{B, A_{s}\right\}$ is also a set of linearly independent columns. Therefore by Fact 2.4, the least-squares problem above has a unique solution.

\subsection{Strict Improvement}

Theorem 2.22

Let $v=B x_{B}$ be a basic weighting corresponding to basis $B$ and let $u=b-v$. Let $v$ be closer to $b$ than $\mu A$, for $\mu \geq 0$ and let $A_{s}$ be linearly independent of the columns of $B$. Let $v_{1}=B x_{B}^{1}+A_{s} x_{s}^{l}$ be the unique least-squares weighting of the problem (2-10), and let $u_{1}=b-v_{1}$. If $u^{T} A_{s}>0$, then the least-squares weighting satisfies $x_{s}^{1}>0$.

Proof: We know $v$ is closer to $b$ than is $\lambda v$ for $\lambda \neq 1$ because $v$ is a basic weighting. We also know $A_{s} \neq \mu v$ because $u^{T} v=0$ by Fact 2.2 , and we know that $u^{T} A_{s}>0$. Let $F=\left\|b-\alpha v-x_{s} A_{s}\right\|^{2}$. Let $\tilde{\alpha}$ and $\tilde{x}_{s}$ be the values of $\alpha$ and $x_{s}$ at the minimum of $F$. From Corollary 2.14, we can conclude that both $\tilde{\alpha}$ and $\tilde{x}_{s}$ are positive. Finally, let $\tilde{v}=\tilde{x}_{s} A_{s}+\tilde{\alpha} v$. Since $v^{1}$ is the least-squares weighting of $\left\{B, A_{s}\right\}$, we have $\left\|b-v^{1}\right\|^{2} \leq\|b-\tilde{v}\|^{2}$.

We need to show that $\|b-\tilde{v}\|^{2} \leq\|b-v\|^{2}$. We know

$$
\begin{aligned}
\|b-v\|^{2} & =b^{T} b-2 v^{T} v+v^{T} v \quad \text { (from Fact 2.2) } \\
& =b^{T} b-v^{T} v .
\end{aligned}
$$

From the proof of Theorem 2.16, we know that

$$
\tilde{x}_{s}=\frac{v^{T} v A_{s}^{T} u}{v^{T} v-\left(A_{s}^{T} v\right)^{2}} \quad \text { and } \quad \tilde{\alpha}=\frac{v^{T} v-A_{s}^{T} v A_{s}^{T} b}{v^{T} v-\left(A_{s}^{T} v\right)^{2}}
$$

and that

$$
\|b-\tilde{v}\|^{2}=b^{T} b-v^{T} v\left(\frac{\left(A_{s}^{T} u\right)^{2}}{v^{T_{v}}-\left(A_{s}^{T} v\right)^{2}}+1\right) .
$$


We know that $A_{s}^{T} u \neq 0$, so $\left(A_{s}^{T} u\right)^{2}>0$. We also know that $v^{T} v-\left(v^{T} A_{s}\right)^{2}>0$ from Fact 2.2. Thus

$$
\frac{\left(A_{s}^{T} u\right)^{2}}{v^{T} v-\left(v^{T} A_{s}\right)^{2}}>0
$$

so that

$$
v^{T} v\left(\frac{\left(A_{s}^{T} u\right)^{2}}{v^{T} v-\left(v^{T} A_{s}\right)^{2}}+1\right)>v^{T} v
$$

Thus

$$
\begin{aligned}
\|b-\tilde{v}\|^{2} & =b^{T} b-v^{T} v\left(\frac{\left(A_{s}^{T} u\right)^{2}}{v^{T} v-\left(v^{T} A_{s}\right)^{2}}+1\right) \\
& <b^{T} b-v^{T} v \\
& =\|b-v\|^{2} .
\end{aligned}
$$

So we have

$$
\left\|b-v^{1}\right\|^{2} \leq\|b-\tilde{v}\|^{2}<\|b-v\|^{2} .
$$

Assume on the contrary that $x_{s}^{1}$ is nonpositive. From Fact 2.5, we know that any convex combination of $\tilde{v}$ and $v^{1}$ is at least as close to $b$ as is $\tilde{v}$. The coefficient of $A_{s}$ is 0 at some combination, since $\tilde{x}_{s}>0$ and we are assuming that $x_{s}^{1} \leq 0$. However, $\tilde{v}$ is strictly closer to $b$ than is $v_{0}$, and if the coefficient of $A_{s}$ is 0 , this convex combination can be no closer to $b$ than $v_{0}$. Thus, somewhere between $v_{1}$ and $\tilde{v}$ we have a solution that is further away from $b$ than is $v_{0}$. This is a contradiction.

\section{Corollary 2.23}

Given the same conditions as in Theorem 2.22, $v^{1}$ is strictly closer to $b$ than is $v$.

Proof: This follows directly from (2-11).

Now consider the following situation. As before, assume we have a basis $B$, a basic weighting $v=B x_{B}$, and $u=b-v$. Now let $A_{s}$ be another column from $A$, where $A_{s}$ is linearly independent of the columns in $B, u^{T} A_{s}>0$, and $v$ is closer to $b$ than is $\mu A_{s}$ for $\mu \geq 0$. The linearly independent set of columns $\left\{B, A_{s}\right\}$, and the solution $\left\{y_{B}^{*}, y_{s}^{*}\right\}$ to

$$
\min \left\|b-B y_{B}-A_{s} y_{s}\right\|^{2}
$$

has the property that $y_{s}^{*}>0$ by Theorem 2.22 . Note however, that we cannot say that $y_{B}^{*}>0$. 


\section{Theorem 2.24}

Given the situation described above, we can find a new basis $\tilde{B}$ where $\tilde{B}$ is formed from a subset of the columns of ( $\left.B A_{s}\right)$, and the solution $\tilde{x}_{B}>0$ has the property that

$$
\min \left\|b-\tilde{B} \tilde{x}_{B}\right\|^{2}<\min \left\|b-B x_{B}\right\|^{2} .
$$

In addition, $A_{s}$ will be one of the columns in $\tilde{B}$.

Proof: Let $y^{*}=\left(\begin{array}{ll}y_{B}^{*} & y_{s}^{*}\end{array}\right)^{T}$ be the solution to

$$
\min \left\|b-\left(\begin{array}{ll}
B & A_{s}
\end{array}\right) y\right\|^{2} .
$$

Because $u^{T} A_{s}>0$, we can use Theorem 2.22 to assert that $y_{s}^{*}>0$. From Corollary 2.23, we also have the fact that

$$
\left\|b-B x_{B}\right\|^{2}>\left\|b-\left(\begin{array}{ll}
B & A_{s}
\end{array}\right) y^{*}\right\|^{2} .
$$

In order to continue, we need some additional notation. We will be forming $\tilde{B}$ from ( $B \quad A_{\theta}$ ), and we may need to iteratively delete a number of the columns of $B$. We will denote the subset of the columns remaining at some iteration by $B^{y}$ (indicating that $B^{y}$ corresponds to the current $y_{B}^{*}$ ). Initially, this means that $B=B^{y}$.

Case 1: $y^{*}>0$

Let $\tilde{B}=\left(\begin{array}{ll}B^{y} & A_{s}\end{array}\right)$ and let $\tilde{x}_{B}=y^{*}$. This assignment is valid, since $y^{*}$ is a basic weighting of the columns of $\left(\begin{array}{ll}B^{y} & A_{s}\end{array}\right)$, and $A_{s}$ is one of the columns of $\tilde{B}$.

Case 2: $y^{*} \geq 0$

Let $\hat{y}^{*}$ be the positive elements of $y^{*}$. Remember that $y_{s}^{*}>0$, so $y_{s}^{*} \in \hat{y}^{*}$. Let $\hat{B}^{y}$ be the columns of $B^{y}$ corresponding to the elements of $\hat{y}^{*}$. Let $\tilde{B}=$ $\left(\begin{array}{ll}\hat{B}^{y} & A_{s}\end{array}\right)$ and let $\tilde{x}_{B}=\hat{y}^{*}$. Again, this is valid because $\hat{y}^{*}$ is a basic weighting of the columns of $\left(\begin{array}{ll}\hat{B}^{y} & A_{s}\end{array}\right)$, and $A_{s}$ is one of the columns of $\tilde{B}$.

Case 3: We do not have $y^{*} \geq 0$.

In this case, we know that at least one element of $y_{B}^{*}$ is negative, and we have a significantly more complicated situation. We need to drop one or more columns from ( $B^{y} A_{s}$ ) such that there exists a positive least-squares solution for the remaining columns. In order to do this, we must redefine $B^{y}$ and $y^{*}$, and then go back and check the sign of $y^{*}$ against Cases 1-3 again. This means that we could perform the steps (to be described) in Case 3 more than once.

The first time through Case 3 , we define $x^{c}=x_{B}$, and we have $B^{y}=B$. (Note that neither equation will be true in subsequent times through Case 3.)

We now form the convex combination

$$
c=\left(\begin{array}{c}
c_{B} \\
c_{s}
\end{array}\right)=\lambda\left(\begin{array}{c}
x^{c} \\
0
\end{array}\right)+(1-\lambda)\left(\begin{array}{c}
y_{B}^{*} \\
y_{s}^{*}
\end{array}\right), x^{c}>0, y_{s}^{*}>0
$$


and seek the smalled $\lambda<1$ such that $c \geq 0$. This $\lambda$ is determined by

$$
\lambda=\max _{y_{j}^{*}<0} \frac{-y_{j}^{*}}{x_{j}^{c}-y_{j}^{*}} .
$$

It is clear for $\lambda \in(0,1)$ that $c_{s}>0$. We will drop all columns of ( $\left.\begin{array}{ll}B^{y} & A_{s}\end{array}\right)$ corresponding to zero elements of $c$.

Each pass through Case 3, one convex combination is formed. In the first pass, we have $B^{y}=B$, and we have proved that $A_{s}$ is retained the first time a convex combination is formed during the column-dropping process. We need to prove that no matter how many convex combinations (and corresponding passes through Case 3) are necessary, $A_{s}$ is not dropped. In order to prove this, we must redefine $x^{c}, B^{y}$ and $y^{*}$ as follows.

Let $\hat{c}$ be the positive elements of $c$. Let $\hat{B}^{y}$ be the columns of $B^{y}$ corresponding to the elements of $\hat{c}$. Let $\hat{y}^{*}$ be the solution to

$$
\min \left\|\boldsymbol{b}-\left(\begin{array}{ll}
\hat{B}^{y} & A_{s}
\end{array}\right) \hat{y}\right\|^{2} .
$$

In order to redefine $x^{c}$ to be $\hat{c}$, we need

$$
\left\|b-B x_{B}\right\|^{2}>\left\|b-\left(\begin{array}{ll}
\hat{B}^{y} & A_{s}
\end{array}\right) \hat{c}\right\|^{2} \geq\left\|b-\left(\begin{array}{ll}
\hat{B}^{y} & A_{s}
\end{array}\right) \hat{y}^{*}\right\|^{2} .
$$

In addition, in order to redefine $y^{*}$ to be $\hat{y}^{*}$ and $B^{y}$ to be $\hat{B}^{y}$, we need

$$
\tilde{y}_{s}^{*}>0 \text {. }
$$

We have

$$
\left\|b-B x_{B}\right\|^{2}>\left\|b-\left(\begin{array}{ll}
B^{y} & A_{s}
\end{array}\right) c\right\|^{2}>\left\|b-\left(\begin{array}{ll}
B^{y} & A_{s}
\end{array}\right) y^{*}\right\|^{2}
$$

from Fact 2.5. We also know that

$$
\left\|b-\left(\begin{array}{ll}
B^{y} & A_{s}
\end{array}\right) c\right\|^{2}=\left\|b-\left(\begin{array}{ll}
\hat{A}^{y} & A_{s}
\end{array}\right) \hat{c}\right\|^{2}
$$

from the definition of $\hat{c}$ and $\hat{A}^{y}$, and since $\hat{y}^{*}$ is a least-squares solution, we have

$$
\left\|b-\left(\begin{array}{ll}
\hat{B}^{y} & A_{s}
\end{array}\right) \hat{c}\right\|^{2} \geq\left\|b-\left(\begin{array}{ll}
\hat{B}^{y} & A_{s}
\end{array}\right) \hat{y}^{*}\right\|^{2} .
$$

Finally, both $\hat{y}^{*}$ and $y^{*}$ are least-squares solutions, but $\hat{B}^{y}$ is a proper subset of $B^{y}$, so

$$
\left\|b-\left(\begin{array}{ll}
\hat{B}^{y} & A_{s}
\end{array}\right) \hat{y}^{*}\right\|^{2} \geq\left\|b-\left(\begin{array}{cc}
B^{y} & A_{s}
\end{array}\right) y^{*}\right\|^{2} .
$$

Altogether, this gives us

$\left\|b-B x_{B}\right\|^{2}>\left\|b-\left(\begin{array}{ll}B^{y} & A_{s}\end{array}\right) \hat{c}\right\|^{2} \geq\left\|b-\left(\begin{array}{ll}\hat{B}^{y} & A_{s}\end{array}\right) \hat{y}^{*}\right\|^{2} \geq\left\|b-\left(\begin{array}{ll}B^{y} & A_{s}\end{array}\right) y^{*}\right\|^{2}$, which proves $(2-12)$. 
Now we need to consider (2-13), the sign of $\hat{y}_{s}^{*}$. We will prove that $\hat{y}_{s}^{*}>0$ by contradiction, so assume that $\hat{y}_{s}^{*} \leq 0$. We will form a convex combination of $y^{*}$ and $\hat{y}^{*}$ as follows. Let $z$ be defined as

$$
z_{j}= \begin{cases}\hat{y}_{j}^{*}, & \text { if } B_{j}^{y} \in \hat{B}^{y} \\ 0, & \text { if } B_{j}^{y} \notin \hat{B}^{y} .\end{cases}
$$

This gives us

$$
\left\|b-\left(\begin{array}{ll}
\hat{B}^{y} & A_{s}
\end{array}\right) \hat{y}^{*}\right\|^{2}=\left\|b-\left(\begin{array}{ll}
B^{y} & A_{s}
\end{array}\right)\left(\begin{array}{c}
z \\
\hat{y}_{s}^{*}
\end{array}\right)\right\|^{2} \geq\left\|b-\left(\begin{array}{ll}
B^{y} & A_{s}
\end{array}\right) y^{*}\right\|^{2} .
$$

Now form the convex combination

$$
c^{z}=\left(\begin{array}{c}
c_{B}^{z} \\
c_{s}^{z}
\end{array}\right)=\lambda\left(\begin{array}{c}
z \\
\hat{y}_{s}^{*}
\end{array}\right)+(1-\lambda)\left(\begin{array}{c}
y_{B}^{*} \\
y_{s}^{*}
\end{array}\right)
$$

Because $\hat{y}_{s}^{*} \leq 0$ and $y_{s}^{*}>0$, we can pick $\lambda \in(0,1]$ such that $c_{s}^{z}=0$. Fact 2.5 says that

$$
\left\|b-\left(\begin{array}{ll}
\hat{B}^{y} & A_{s}
\end{array}\right) \hat{y}^{*}\right\|^{2} \geq\left\|b-\left(\begin{array}{ll}
\hat{B}^{y} & A_{s}
\end{array}\right) c^{z}\right\|^{2} \geq\left\|b-\left(\begin{array}{ll}
B^{y} & A_{s}
\end{array}\right) y^{*}\right\|^{2} .
$$

However, this would imply that

$$
\left\|b-B x_{B}\right\|^{2}>\left\|b-\left(\begin{array}{ll}
\hat{B}^{y} & A_{s}
\end{array}\right) c^{2}\right\|^{2},
$$

which when $c_{s}^{z}=0$, can be rewritten as

$$
\left\|b-B x_{B}\right\|^{2}>\left\|b-\left(\begin{array}{ll}
\hat{B}^{y} & A_{s}
\end{array}\right)\left(\begin{array}{c}
c_{B}^{z} \\
c_{s}^{z}
\end{array}\right)\right\|^{2}=\left\|b-\left(\begin{array}{ll}
\hat{B}^{y} & A_{s}
\end{array}\right)\left(\begin{array}{c}
c_{B}^{z} \\
0
\end{array}\right)\right\|^{2},
$$

and thus

$$
\left\|b-B x_{B}\right\|^{2}>\left\|b-\hat{B}^{y} c_{B}^{z}\right\|^{2} .
$$

However, $\hat{B}^{y}$ is a proper subset of the columns of $B$, and $x_{B}$ is the least-squares solution to

$$
\min \left\|b-B x_{B}\right\|^{2}
$$

so that we know

$$
\left\|b-B x_{B}\right\|^{2} \leq\left\|b-\hat{B}^{y} c_{B}^{z}\right\|^{2} .
$$

This is our contradiction. Thus we must have $\hat{y}_{s}^{*}>0$, and (2-13) has been shown to be true. We can therefore set $x^{c}=\hat{c}, B^{y}=\hat{B}^{y}, y^{*}=\hat{y}^{*}$, and go back and check this new $y^{*}$ against Cases 1-3. Note that if Case 3 again applies and additional columns must be dropped, we will have the following upon entry:

$$
\left\|b-B x_{B}\right\|^{2}>\left\|b-\left(\begin{array}{ll}
B^{y} & A_{s}
\end{array}\right) x^{c}\right\|^{2} \geq\left\|b-\left(\begin{array}{ll}
B^{y} & A_{s}
\end{array}\right) y^{*}\right\|^{2},
$$


and when a new convex combination $c$ is formed, we will find

$$
\left\|b-B x_{B}\right\|^{2}>\left\|b-\left(\begin{array}{ll}
B^{y} & A_{s}
\end{array}\right) x^{c}\right\|^{2}>\left\|b-\left(\begin{array}{ll}
B^{y} & A_{s}
\end{array}\right) c\right\|^{2}>\left\|b-\left(\begin{array}{ll}
B^{y} & A_{s}
\end{array}\right) y^{*}\right\|^{2}
$$

by Fact 2.5. Thus (2-12) will still hold in subsequent passes through Case 3. We repeat this process of checking the sign of $y^{*}$ and dropping columns until Case 1 or Case 2 is satisfied.

We will now show that $y^{*}$ will eventually satisfy Case 1 or Case 2 . We know that $y_{s}^{*}>0$, no matter how many convex combinations are formed, and no matter how many times $y^{*}$ and $B^{y}$ are consequently redefined. Therefore column $A_{s}$ is always retained during this process. Thus we can conclude that in the worst case, we will find $y^{*}>0$ when $B^{y}=\emptyset$ (and $A_{s}$ is the only remaining column in $\tilde{B})$, at which time Case 1 is satisfied.

Note that Theorem 2.24 did not disallow the possibility that all columns be dropped from $A^{y}$ before we can find a $y^{*}>0$. That is, it implies that it could be possible that $\left\{A_{k}\right\}=A_{s}$ and $x^{1}=y_{s}^{*}$. We will prove that this is actually not possible.

\section{Corollary 2.25}

If the first column ever added to $B$ is $A_{r}$ where $r$ is determined by (2-6) in Theorem 2.15, then $y^{*}$ will satisfy Case 1 or 2 before all columns other than $A_{s}$ are dropped from the basis.

Proof: Assume that $y^{*}$ never satisfies Case 1 or Case 2 until only column $A_{s}$ is remaining (and $y^{*}=y_{s}^{*}>0$ ). From Theorem 2.24, we know that

$$
\left\|b-B x_{B}\right\|^{2}>\left\|b-\tilde{B} \tilde{x}_{B}\right\|^{2},
$$

which (if all columns other than $A_{s}$ are dropped) can be rewritten as

$$
\left\|b-B x_{B}\right\|^{2}>\left\|b-A_{a} y_{s}^{*}\right\|^{2} .
$$

Since $A_{r}$ was the first column placed in $B$, we know from Theorem 2.24 that

$$
\left\|b-A_{r} y_{r}^{*}\right\|^{2}>\left\|b-B x_{B}\right\|^{2},
$$

where $y_{r}^{*}>0$ is the solution to

$$
\min \left\|b-A_{r} y_{r}\right\|^{2}
$$

Thus

which implies

$$
\left\|b-A_{r} y_{r}^{*}\right\|^{2}>\left\|b-B x_{B}\right\|^{2}>\left\|b-A_{s} y_{s}^{*}\right\|^{2},
$$

$$
\left\|b-A_{r} y_{r}^{*}\right\|^{2}>\left\|b-A_{s} y_{s}^{*}\right\|^{2} .
$$

However, $A_{r}$ was chosen to be the solution to (2-5) (see Theorem 2.15). Thus we have a contradiction. 


\subsection{Infeasibility}

Until now, we have not considered the feasibility or infeasibility of (2-1). This section discusses the issue of infeasibility and how it affects the solution to the least-squares problems considered in Theorems 2.15 and 2.16, as well as other related issues.

Fact 2.26

If $A^{T} b \leq 0$, we can conclude that (2-1) is infeasible.

Proof: Given that $b \neq 0$, this follows directly from Farkas' Lemma.

Fact 2.26 implies that if in the process of finding the solution to (2-5) (see Theorem 2.15), we find that $A_{j}^{T} b \leq 0 \forall j$, then we have established that (2-1) is infeasible.

\section{Fact 2.27}

Let $A$ be as in Fact 2.26. Let $v \neq 0$ be closer to $b$ than any $\lambda v, \lambda \neq 1$, and let $u=b-v$. If $u \neq 0$ and $u^{T} A \leq 0$, then the original problem (2-1) is infeasible.

Proof: We know from Fact 2.2 that $b^{T} u=u^{T} u$, and we know that $u \neq 0$. Therefore this follows directly from Farkas' Lemma.

\section{Corollary 2.28}

Let $v$ be a basic weighting corresponding to basis $B$ and let $u=b-v$. If $u^{T} A_{j} \leq 0 \forall A_{j} \notin B$, then (2-1) is infeasible.

Proof: We know that $v$ is a basic weighting of $B$, so we can conclude from Theorem 2.18 that $u^{T} A_{j}=0 \forall A_{j} \in B$. Thus we have $u^{T} A_{j} \leq 0 \forall A_{j}$. We can see by Fact 2.27 that $(2-1)$ is thus infeasible.

\section{Theorem 2.29}

Let $v=B x_{B}$ be a basic weighting corresponding to the basis $B$ and let $u=b-v$. If infeasibility is detected (i.e., if $u^{T} A_{j} \leq 0 \forall A_{j} \notin B$ ), then $v$ is at least as close to $b$ as is any other nonnegative weighting of the columns of $A$.

Proof: Let $B I$ be the set of indices such that $j \in B I$ if and only if $A_{j} \in B$. We can write $v$ as

$$
v=\sum_{j \in B I} A_{j}\left(x_{B}\right)_{j}
$$

where $\left(x_{B}\right)_{j}$ is the component of $x_{B}$ corresponding to $A_{j}$.

Since we have discovered infeasibility, we know that $u \neq 0$. We also know that $v$ is closer to $b$ than is any $\lambda v_{B}, \lambda \neq 1$ because $x_{B}$ is the least-squares solution to

$$
\min \left\|b-\sum_{j \in B I} A_{j}\left(x_{B}\right)_{j}\right\|^{2} .
$$


We therefore know:

$$
\begin{aligned}
u^{T} b & =u^{T} u \text { by Fact } 2.2 \\
u^{T} A_{j} & =0 \forall j \in B I \text { by Theorem } 2.18 \\
u^{T} A_{j} & \leq 0 \forall j \notin B I \text { because infeasibility has been detected. }
\end{aligned}
$$

Let

$$
\tilde{v}=\sum_{j=1}^{n} A_{j} \tilde{x}_{j}
$$

be any other nonnegative weighting of the columns of $A$. Therefore we have $\tilde{x}_{j} \geq 0 \forall j$. Let $\tilde{u}=b-\tilde{v}$. Consider

$$
\begin{aligned}
u^{T} \tilde{u}_{B} & =u^{T} b-u^{T} \sum_{j=1}^{n} A_{j} \tilde{x}_{j} \\
& =u^{T} u-\sum_{j \in B I} u^{T} A_{j} \tilde{x}_{j}-\sum_{j \notin B B I} u^{T} A_{j} \tilde{x}_{j} \\
& =u^{T} u-\sum_{j \notin B I} u^{T} A_{j} \tilde{x}_{j} \geq u^{T} u
\end{aligned}
$$

Now consider

$$
u^{T} u=\|u\|\|u\| ; \quad u^{T} \tilde{u}=\|u\|\|\tilde{u}\| \cos \theta,
$$

where $\theta$ is the angle between $u$ and $\tilde{u}$. We have

$$
\|u\|\|\tilde{u}\| \cos \theta \geq\|u\|\|u\| \Rightarrow\|\tilde{u}\|\|\tilde{u}\| \cos \theta \geq\|u\|\|\tilde{u}\|,
$$

which can be extended to

$$
\|\tilde{u}\|\|\tilde{u}\| \geq\|\tilde{u}\|\|\tilde{u}\| \cos \theta \geq\|u\|\|\tilde{u}\| \geq\|u\|\|\tilde{u}\| \cos \theta .
$$

Therefore $\tilde{u}^{T} \tilde{u} \geq u^{T} \tilde{u} \geq u^{T} u$. So we can conclude that $v$ is at least as close to $b$ as is any other nonnegative weighting of the columns of $A$.

\section{Corollary 2.30}

Given $B, v$ and $u$ as in Theorem 2.29. If $v$ is closer to $b$ than is any other nonnegative weighting of the columns of $A$, then infeasibility will be detected (that is, we will find $u^{T} A_{j} \leq 0 \forall j \notin B$ ).

Proof: Assume that $u^{T} A_{s}>0$ for some $A_{s} \notin B$. If we add $A_{s}$ to $B$ and drop columns as described in Section 2.6, then we will find a $\tilde{B}$ and $\tilde{x}_{B}>0$ such that $\tilde{v}=\tilde{B} \tilde{x}_{B}$ is closer to $b$ than is $v$. This contradicts the assumption that $v$ is closer to $b$ than is any other nonnegative weighting of the columns of $A$. 


\subsection{The Least-Squares Phase I Algorithm}

As stated in Section 2.1, the main objective of the Phase $I$ algorithm to be presented here is to build a basis matrix, made up of selected columns of $A$, and to find positive weightings of these columns such that the basis and the associated positive weightings are a solution to (2-2). Remember that we have rescaled (2-1) such that $\left\|A_{j}\right\|=1 \forall j$ and $\|b\|=1$.

At the start of an iteration, we are given a basis $B$, composed of a linearly independent subset of the columns of $A$, with the property that the least-squares weighting of these columns which yields the closest approximation to the righthand side $b$ is a positive weighting. The iterative step tests whether the current approximation is the closest approximation with a positive weighting, and if not, selects an incoming column with the property that a positive combination of the previous approximation and the incoming column is a strictly better approximation to the right-hand side. There exists, however, a (possibly proper) subset of the columns of the augmented basis such that the least-squares weighting is positive, and is also a better approximation to the right-hand side than the simple positive combination. This subset of the augmented set of basic columns can be used to start the next iteration.

\section{Algorithm Pseudocode}

Notation and Variables:

$A$ : The original $m \times n$ matrix of $(2-1)$.

$b$ : The original right-hand side of (2-1).

$n b i$ : The number of currently basic columns (the number of columns in $B$ ).

$B$ : The matrix of currently basic columns. $m \times n b i$ of $B$ is used, and $n b i$ can be as large as $m$.

$B I:$ An $m$-vector containing indices of the columns of $B$ relating them to the columns of $A$. For example, if $B I[i]=j$, the $i$ th column of $B$ contains the $j$ th column of $A . B I[i]=0$ means no column corresponds to the $i$ th component of $B I$, which implies there must be less than $i$ basic columrs.

$x_{B}$ : The current positive weightings of the columns of $B$. The first $n b i$ components of $x_{B}$ (corresponding to the nbi columns in $B$ ) are used, and $n b i$ can be as large as $m$.

$v$ : The current approximation $B x_{B}$.

$u$ : The current residual $b-v$.

$y$ : The current least-squares solution of $\min \|b-B y\|^{2}$. The first nbi components of $y$ are used, and $n b i$ can be as large as $m$. 
$X$ : The vector to hold the solution to $(2-1)$. Note that $x_{B}$ holds the solution to $(2-2)$.

0 . Initialization \{no columns initially in basis\}

$B:=\emptyset \quad$ \{no columns in basis\}

$x_{B}:=\emptyset \quad$ \{no current basic solution

$B I:=\emptyset$ nc basic indices

$n b i:=0 \quad\{$ set number of basic columns to 0$\}$

$u:=b \quad\{$ set residual to $b\}$

$v:=0 \quad$ set current approximation $v=B x_{B}=0$ \}

$X:=0 \quad$ sset final solution to 0$\}$

1. Stariup \{find the first column to place in $B$ \}

If $b^{T} A_{j} \leq 0 \forall j=1, \ldots, n$ then

Go to 4 . \{the problem is infeasible

$s:=\operatorname{argmax}_{j: b^{T} A_{j}>0} b^{T} A_{j}$

Place $A_{s}$ in the first column of $B$.

$B I[1]:=s \quad$ \{record first column

$y[1]:=b^{T} A_{s} \quad$ \{record basic solution

$n b i:=1 \quad$ \{set number of baxic columns\}

2. Main Loop \{Add columns to $B$ until $u=0$ or infeas. is discovered.

$x_{B}:=y$

$v:=B x_{B} \quad$ \{set current approximation $\}$

$u:=b-v \quad$ \{set current residual $\}$

If $u=0$ then

Go to 4 . \{solution found\}

If $u^{T} A_{j} \leq 0 \quad \forall j \notin B I$ then

Go to 4 . $\quad$ infeasibility discovered

$n b i:=n b i+1 \quad$ increment nurnber of basic columns\}

$$
s=\underset{\substack{j \in B I \\ j, A_{j}^{T} v>0}}{\operatorname{argmax}} \frac{A_{j}^{T} u}{\left(v^{T} v-\left(A_{j}^{T} v\right)^{2}\right)^{1 / 2}}
$$

Place $A_{s}$ in column $n b i$ of $B$.

$B I[n b i]:=s \quad$ \{record position of incoming column

Set $c=\left(\begin{array}{c}x_{B} \\ 0\end{array}\right)$

3. Least-Squares Loop

Solve $\|B y-b\|^{2}$ for $y$

If $y>0$ then

Go to 2. \{new $B$ and $x_{B}$ found; repeat Main Loop

If $y \geq 0$ then 
Remove all columns from $B$ and elements from $x_{B}$ that correspond to zero components of $y$. Update $B I$ accordingly.

Go to 2. \{new $B$ and $x_{B}$ found; repeat Main Loop

$\lambda:=\max _{y_{i}<0}-y_{i} /\left(c_{i}-y_{i}\right)$

$c:=\lambda c+(1-\lambda) y \quad$ form convex combination of $c$ and $y$ \}

Remove all coluinns from $B$ and elements from $x_{B}$ and $c$ that

correspond to zero components of $c$. Update $B I$ accordingly.

Go to 3. \{repeat Least-Squares loop\}

4. Done \{clean up\}

for $i:=1$ to $n b i$ do $\quad$ form the answer to the original problern\}

$X_{B I[i]}:=x_{B}[i]$

Report on feasibility and output the solution found.

We now : aake the following observations:

1. The approximation $v=B x_{B}$ is strictly closer to $b$ at each iteration (see Theorem 2.24). This means that degeneracy does not cause problems; cycling cannot occur, and the process terminates in a finite number of steps because given $B, x_{B}$ is uniquely determined, and there are a finite number of bases $B$.

2. If infeasibility is detected, then the current approximation $v=B x_{B}$ is closer to $b$ than is any other nonnegative weighting of the columns of $A$ (see Theorem 2.29). Similarly, if $u \neq 0$ and $v=B x_{B}$ is closer to $b$ than is any other nonnegative weighting of the columns of $A$, then infeasibility will be detected when trying to add a new column (sue Corollary 2.30).

3. Whenever a new column is chosen from $A$ to enter $B$, it is linearly independent of the columns currently in $B$ (see Corollary 2.19). Also due to the linear independence of entering columns, our least-squares solution (the solution to $\min \|b-\tilde{B} y\|$ ) is always unique, so numerical difficulties aside, rank deficient least-squares problems are never encountered.

\subsection{Variations}

\section{Free Variables}

Consider the system

$$
\begin{aligned}
A x & =b \\
x_{i} \geq 0 & \text { for some set of } i \\
x_{j} & \text { free } \quad \text { for some set of } j
\end{aligned}
$$

This system could always be converted to the form of (2-1) by replacing each free variable $x_{j}$ with two new nonnegative variables $x_{j}^{\prime}$ and $x_{j}^{\prime \prime}$ where the original $x_{j}$ 
is now represented by $x_{j}^{\prime}-x_{j}^{\prime \prime}$. However, let us consider how to solve a feasibility problem with free variables without actually adding any more variables.

In the pure algorithm described in Section 2.8, the entering columns is $A_{s}$ where $s$ is determined by (2-8). This assumes that all variables are nonnegative. If free variables are present, we can write the rule for selecting the incoming column $s$ as

$$
s=\operatorname{argmax}\left\{\max _{\substack{i, x_{i} \geq 0 \\ i: A_{i}^{T} \cup>0}} \frac{A_{i}^{T} u}{\left(v^{T} v-\left(A_{i}^{T} v\right)^{2}\right)^{1 / 2}}, \max _{\substack{j, x_{j} \text { Ireo } \\ j,\left|A_{j}^{T} w\right|>0}} \frac{\left|A_{j}^{T} u\right|}{\left(v^{T} v-\left(A_{j}^{T} v\right)^{2}\right)^{1 / 2}}\right\} .
$$

Therefore we see that we can select an incoming column when free variables are present without actually adding new variables and columns. Infeasibility is detected when $A_{i}^{T} u \leq 0, \forall i: x_{i} \geq 0$, and $A_{j}^{T} u=0 \quad \forall j: x_{j}$ is free.

The only other change in the algorithm concerns testing the sign of $y$ in the Least-Squares Loop part of the algorithm. Elements of $y$ corresponding to free variables may take on any nonzero value. If such an eler.ent of $y$ is found to be zero, it is dropped just as any other zero element of $y$ would be. The only components of $y$ that must be strictly positive are those corresponding to variables restricted to be nonnegative. Thus, when $\lambda$ is formed, the only components $y_{i}<0$ that are considered are those corresponding to nonnegative variables.

All the results previously discussed (strict improvement, infeasibility detection, etc.) still hold, as these m ifications to the pure algorithm simply implement the result of spliting free variables without actually doing the work of adding variables and columns.

\section{No Denominator}

Consider the selection of the incoming columns not currently in the basis, an 0 . As the denominator is never dire infeasibility, strict improvement, e with 1 , we produce a perfectly vi computation per candidate column

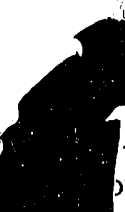

$A_{s}$. We must form $A_{j}^{T} u$ for all or all columns such that $A_{j}^{T} u>$ rulany of the proofs relating to ce the denominator of $(2-8)$
rection rule that requires less

It would be expected that this modified might not perform as well as the pure rule, since we may not be selecting the $A_{s}$ that minimizes (2-7). However, we can still guarantee $(\alpha, \beta)>0$ because, $T, u>0$.

As it turns out, the results produced (on test problems to be described later) show very little difference in iteration count between either of these two variations ("no denominator" and "iree variable") and the pure algorithm. Apparently, it is the direction of the candidate incoming column that is important, not the particular scaling fartor used in the column-selection rule. 


\section{Crash Basis}

Until now, we have built up the basis $B$ starting from scratch-that is, initially $B$ contains no columns. If we could pick out a starting set of linearly independent columns without much effort, we could start with them already in $B$.

Consider multiplying by -1 all rows of $A$ (and elements of $b$ ) corresponding to negative elements of $b$. This gives us a positive right-hand side. Now consider selecting all columns of this modified $A$ that are columns of the identity matrix, i.e., columns of the form $e_{j}$, where $e$ is a vector of zeros with a 1 in the $j$ th position. (Note that most columns of this form will correspond to slack variables.) If we place all such columns in $B$, and elirinate duplicate columns, we will get the problem $\min \|b-B x\|$, and the solution $x_{B}$ will consist of the elements of $b$ corresponding to the nonzero elements of the identity columns.

All of the theory for the pure algorithm holds for this method as well, with the exception of Corollary 2.25. Since we do not know that the column $A_{s}$ satisfying (2-5) was the first column selected to enter the basis, we can no longer guarantee that all columns (other than the incoming column) will not be dropped. In our computational experience, such dropping of all columns has not been observed, and is thus presumed to be rare in practice.

\subsection{Discussion}

We have proved in Theorem 2.29 and Corollary 2.30 that if $(2-1)$ is infeasible, we will detect such infeasibility when our current solution $x_{B}>0$ is as close as possible to the right-hand side $b$. That is, $v=B x_{B}$ is at least as close to $b$ as is any other nonnegative weighting of the columns of $A$. Thus we see that the least-squares algorithm actually solves the problem

$$
\begin{aligned}
\min & \frac{1}{2}\|b-A x\|^{2} \\
\text { s.t. } & x \geq 0 .
\end{aligned}
$$

If the least-squares algorithm finds a feasible solution to (2-1), then the optimal objective value of (2-15) is 0 . Otherwise, the optimal objective of (2-15) is positive, we have an optimal solution $x_{B}$, and no feasible solution exists to (21).

Problem (2-15) is often called the "non-negative least-squares" problem (NNLS), and can also be considered a degenerate case of the "bounded leastsquares" problem (BLS), in which $x$ can have both upper and lower bounds. Lawson and Hanson [12] present an algorithm for NNLS that is very closely related to our algorithm. The only differences between the algorithms is that first, a denominator of " 1 " is used in the column selection rule of NNLS. Secondly, after an incoming column $A$, has been successfully introduced into the basis (and all necessary dropping of other basic colurnns is complete), our algorithm takes all basic variables with a zero value and makes them nonbasic, while Lawson and Hanson's NNLS algorithm does not. 
We implemented both the NNLS algorithm and the "denominator-of-1" variation of the least-squares algorithm, and tested the efficiency of the algorithms on the smallest 30 linear programming problems available from NETLIB. (See [7] and Section 3 for more details about these problems.) In practice, these two algorithms rarely chose different incoming columns. A substantial difference between the two algorithms was observed only once, on problem SCSD6, which is highly degenerate in the simplex method sense. It also appears to be the case (in practice) that the NNLS algorithm will occasionally have the same final basic index set as does the least-squares algorithm with an additional handful of basic variables. In our experiments, these additional basic variables were all at a very low level (on the order of $10^{-10}$ or less). Thus the least-squares algorithm occasionally found a "cleaner" solution than did the NNLS algorithm, apparently due to numerical error.

Björck [3] presents an algorithm for BLS, that when applied to NNLS, is even closer to our algorithm that is Lawson and Hanson's algorithm for NNLS. The only difference between the BLS algorithm and the least-squares algorithm is that a denominator of " 1 " is used in the column selection rule of NNLS. Therefore, the "denominator-of-1" version of the least-squares algorithm is equivalent to the BLS algorithm. Neither [3] nor [12] give any numerical results for this algorithm.

We also found the following interesting equivalence between the least-squares alg, rithm and the convex quadratic programming algorithm due to Dantzig [5] and Van de Panne \& Whinston [14]. Before we can show this equivalence, we must first reformulate (2-15) as a quadratic programming problem.

The constrained least-squares (2-15) has the following necessary and sufficient conditions for optimality:

$$
\begin{aligned}
-A^{T} b+A^{T} A x & \geq 0 \\
x & \geq 0 \\
x^{T}\left(-A^{T} b+A^{T} A x\right) & =0 .
\end{aligned}
$$

These conditions can be formulated as the Linear Complementarity Problem (LCP)

$$
\begin{aligned}
q+M x & \geq 0 \\
x & \geq 0 \\
x^{T}(q+M x) & =0
\end{aligned}
$$

where $q=-A^{T} b$ and $M=A^{T} A$. Because this $M$ is $-y m m e t r i c$ and positive semi-definite, this LCP is equivalent to the quadratic 1 rogram

$$
\begin{array}{ll}
\min & q^{T} x+\frac{1}{2} x^{T} M x=c \\
\text { s.t. } & \therefore \geq 0 .
\end{array}
$$


(This problem could, of course, be derived directly from (2-15).)

Accordingly, any convex quadratic programming algorithm would be applicable to (2-17). Although we are going to demonstrate an equivalence between the quadratic programming algorithm developed by Dantzig and by Van de Panne \& Whinston, the very same algorithmic ideas are developed by Goldfarb \& Idnani [10] from an active-set point of view. (The following description of this algorithm is adapted from the book by Cottle, Pang and Stone [4].)

\section{The Quadratic Programming Algorithm}

The algorithm is based on principal pivotal transforms of the system $y=q+M x$, which can be written in tableau form as

$$
\begin{array}{c|c|c|}
\multicolumn{1}{c}{} & \multicolumn{1}{c}{1} & \multicolumn{1}{c}{x} \\
\cline { 2 - 3 } 2 c & 0 & q^{7} \\
\cline { 2 - 3 } y & q & M \\
\cline { 2 - 3 } & &
\end{array}
$$

where $c$ is as in (2-17). At any point in the algorithm, we will have two index sets $\alpha$ and $\beta$, corresponding to basic and nonbasic $x$ variables, respectively. Thus if we partition and relabel the original tableau as

\begin{tabular}{l|c|c|c|}
\multicolumn{2}{c}{1} & \multicolumn{1}{c}{$x_{\beta}$} & \multicolumn{1}{c}{$y_{\alpha}$} \\
\cline { 2 - 4 } $2 c$ & 0 & $q_{\beta}^{T}$ & $q_{\alpha}^{T}$ \\
\cline { 2 - 4 }$y_{\beta}$ & $q_{\beta}$ & $M_{\beta \beta}$ & $M_{\beta \alpha}$ \\
\cline { 2 - 4 }$x_{\alpha}$ & $q_{\alpha}$ & $M_{\alpha \beta}$ & $M_{\alpha \alpha}$ \\
\cline { 2 - 4 } & &
\end{tabular}

then we can write a general tableau during the algorithm in terms of the original tableau as

\begin{tabular}{c|c|c|c|}
\multicolumn{1}{c}{$2 c$} & \multicolumn{2}{c}{$x_{\beta}$} & $y_{\alpha}$ \\
\cline { 2 - 4 }$y_{\beta}$ & $-q_{\alpha}^{T} M_{\alpha \alpha}^{-1} q_{\alpha}$ & $q_{\beta}^{T}-q_{\alpha}^{T} M_{\alpha \alpha}^{-1} M_{\alpha \beta}$ & $q_{\alpha}^{T} M_{\alpha \alpha}^{-1}$ \\
\cline { 2 - 4 }$x_{\alpha}$ & $q_{\beta}-M_{\beta \alpha} M_{\alpha \alpha}^{-1} q_{\alpha}$ & $M_{\beta \beta}-M_{\beta \alpha} M_{\alpha \alpha}^{-1} M_{\alpha \beta}$ & $M_{\beta} \alpha M_{\alpha \alpha}^{-1}$ \\
\cline { 2 - 4 } & $-M_{\alpha \alpha}^{-1} q_{\alpha}$ & $-M_{\alpha \alpha}^{-1} M_{\alpha \beta}$ & $M_{\alpha \alpha}^{-1}$ \\
\cline { 2 - 4 }
\end{tabular}

From this tableau, we can see that when the nonbasic variables $x_{\beta}$ nd $y_{\alpha}$ are set to 0 , the basic variables $x_{\alpha}$ and $y_{\beta}$ can be expressed as

$$
\begin{aligned}
& x_{\alpha}=-M_{\alpha \alpha}^{-1} q_{\alpha} \\
& y_{\beta}=q_{\beta}-M_{\beta \alpha} M_{\alpha \alpha}^{-1} q_{\alpha} .
\end{aligned}
$$

We will rewrite the general tableau (after $k$ principal pivots) as 


\begin{tabular}{c|c|c|c|}
\multicolumn{3}{c}{} & \multicolumn{3}{c}{$x_{\beta}^{k}$} & \multicolumn{1}{c}{$y_{\alpha}^{k}$} \\
\cline { 2 - 4 } $2 c$ & $\theta^{k}$ & $\left(q_{\beta}^{k}\right)^{T}$ & $\left(q_{\alpha}^{k}\right)^{k}$ \\
\cline { 2 - 4 }$y_{\beta}^{k}$ & $q_{\beta}^{k}$ & $M_{\beta \beta}^{k}$ & $M_{\beta \alpha}^{k}$ \\
\cline { 2 - 4 }$x_{\alpha}^{k}$ & $\boldsymbol{q}_{\alpha}^{k}$ & $M_{\alpha \beta}^{k}$ & $M_{\alpha \alpha}^{k}$ \\
\cline { 2 - 4 } & &
\end{tabular}

in order to ease the notation when examining the algorithm in more detail. We now describe the quadratic programming algorithm as applied to (2-17) in pseudocode.

0 . Initialization.

$$
\begin{aligned}
& \left(q^{0}, M^{0}\right):=(q, M) \\
& k:=0 \\
& \alpha:=\emptyset \\
& \beta:=\{1, \ldots, n\}
\end{aligned}
$$

1. Check stopping conditions.

$s:=\operatorname{argmin}\left\{q_{i}^{k}: i \in \beta\right\}$

If $q_{s}^{k} \geq 0$, the solution is $x_{\alpha}=q_{\alpha}^{k}, x_{\beta}=0$.

If $q_{s}^{k}<0$, choose $x_{s}^{k}$ as the incoming variable.

Note that we do not need to check for infeasibility here, as (2-17) always has a solution.

2. Determine outgoing (blocking) basic $x$ variables, if any.

Let

$$
\gamma=\min _{i:\left(\left(M_{\alpha \beta}^{k}\right)_{0}\right)_{1}<0} \frac{\left(q_{\alpha}^{k}\right)_{i}}{-\left(\left(M_{\alpha \beta}^{k}\right)_{s}\right)_{i}}
$$

where $\left(M_{\alpha \beta}^{k}\right)$, is the $s$ th column of $M_{\alpha \beta}^{k}$,

$\left(\left(M_{\alpha \beta}^{k}\right)_{i}\right)_{i}$ is the $i$ th component of that column, and similarly $\left(q_{\alpha}^{k}\right)_{i}$ is the $i$ th component of $q_{\alpha}^{k}$.

3. Perform a pivot.

If

$$
\gamma \geq \frac{\left(q_{\beta}^{k}\right)_{i}}{-\left(\left(M_{\beta \beta}^{k}\right)_{s}\right)_{i}}
$$

then there is no outgoing basic $x$ variable. In this case,

Move index $s$ from index set $\beta$ to $\alpha$.

Set $k=k+1$.

Go to Step 1 .

Otherwise, let $r$ be the index corresponding to the minimum ratio $\gamma$

Move index $r$ from index set $\alpha$ to $\beta$.

Set $k=k+1$.

Go to Step 2. 
Further discussion of this algorithm can be found in Cottle, Pang \& Stone [4].

We now demonstrate the equivalences between this algorithm and the nodenominator version of the least-squares algorithm. Let us assume that at some point in both algorithms we have the same index sets $\alpha$ and $\beta$ corresponding to basic and nonbasic $x$ variables, respectively. We can rewrite the partitioned original tableau in terms of our least-squares algorithm as

\begin{tabular}{|c|c|c|c|}
\hline & 1 & $x_{\beta}$ & $y_{\alpha}$ \\
\hline $2 c$ & 0 & $-b^{T} \bar{N}$ & $-b^{T} B$ \\
\hline & $-N^{T} b$ & $N^{T} N$ & $N^{T} B$ \\
\hline & $-B^{T} b$ & $\bar{B}^{T} \bar{N}$ & $B^{T} \bar{B}$ \\
\hline
\end{tabular}

Note that $q_{\beta}=-N^{T} b$, and so on. Similarly, we rewrite the general tableau as

\begin{tabular}{|c|c|c|c|}
\hline & 1 & $x_{\beta}$ & $y_{0}$ \\
\hline & $-b^{T} B\left(B^{T} B\right)^{-1} B^{T} b$ & $-b^{T} N+b^{T} B\left(B^{T} B\right)^{-1} B^{T} N$ & $-b^{T} B\left(B^{T} \bar{B}\right)^{-T}$ \\
\hline & $-N^{T} b+N^{T} B\left(B^{T} B\right)^{-1} B^{T} b$ & $N^{T} N-N^{T} B\left(B^{T} B\right)^{-1} B^{T} N$ & $N^{T} B\left(B^{T} B\right)^{-1}$ \\
\hline & $\left(B^{T} \bar{B}\right)^{-1} B^{T} b$ & $-\left(B^{T} B\right)^{-1} B^{T} N$ & $\left(B^{T} B\right)^{-}$ \\
\hline
\end{tabular}

Thus we see that $x_{\alpha}=-M_{\alpha \alpha}^{-1} q_{\alpha}=\left(B^{T} B\right)^{-1} B^{T} b=x_{B}$, and the two solutions are the same. Therefore, given the same index set of basic $x$ variables, both the quadratic programming algorithm and the least-squares algorithm produce the same solution $x_{\alpha}=x_{B}$.

To further compare the two algorithms, assume that again both algorithms have the same index sets $\alpha$ and $\beta$ corresponding to the basic and nonbasic $x$ variables. We will consider which nonbasic $x$ variable is chosen to become basic in both algorithms. where

In the quadratic programming algorithm we select incoming variable $x_{s}$,

$$
\begin{aligned}
s & =\underset{i \in \beta}{\operatorname{argmin}}\left\{q_{i}^{k}\right\}, \\
& =\underset{i \in \beta}{\operatorname{argmin}}\left\{\left(q_{\beta}-M_{\beta \alpha} M_{\alpha \alpha}^{-1} q_{\alpha}\right)_{i}\right\} \\
& =\underset{i \in \beta}{\operatorname{argmin}}\left\{-N_{i}^{T} b+N_{i}^{T} B x_{B}\right\} \\
& =\underset{i \in \beta}{\operatorname{argmin}}\left\{-N_{i}^{T} u\right\} .
\end{aligned}
$$

If we find that $q_{s}^{k}=-N_{s}^{T} u \geq 0$, then in the quadratic programming algorithm, we know we have a solution to (2-17). Similarly, if $N_{s}^{T} u \leq 0$, the least-squares algorithrn has either found a solution to (2-1) (in which case $u=0$ ), or has detected infeasibility of (2-1). In either case, the least-squares algorithm has 
found a solution to (2-17). Thus $N_{s}^{T} u \leq 0$ is the termination condition for both algorithms. (Note that the quadratic programming algorithm never finds infeasibility, as (2-17) always has a solution.) On the other hand, if $q_{s}^{k}=$ $-N_{s}^{T} u<0$, then $x_{s}$ is the incoming variable for both problems. Thus the two algorithms have the same termination conditions and the same column-selection rules.

Given that we start an iteration with the same index sets, and that the same incoming variable $x_{s}$ is selected, we will now consider how the two algorithms proceed. In the least-squares algorithm, $x_{s}$ simply becomes basic, even though this could cause other basic variables to become negative. However, the quadratic programming algorithm checks for and pivots out all (if any) variables which would become negative, only then making $x_{s}$ basic. If there is no outgoing basic variable, then both algorithms add $x_{s}$ to the set of basic variables, all other basic variables remain nonnegative, and the algorithms again share the same index sets.

Assume now that bringing $x_{s}$ into the set of basic $x$ variables will cause at least one of the other basic variables to become negative. In the case of the leastsquares algorithm, this situation means that at least one of the previously basic $x$ variables is nonnegative, and at least one of these negative basic variables must be dropped from the basis. Similarly, the quadratic programming algorithm must find and eliminate from the basis at least one outgoing (blocking) variable. We will now demonstrate that given the same index sets, and the same incoming variable $x_{s}$, if a column must be dropped from the basis in order to maintain nonnegativity of the basic $x$ variables, both algorithms will select the same basic $x$ variable to be removed from the basis.

The quadratic programming algorithm will select $x_{r}$ to become nonbasic, where

$$
\begin{aligned}
r= & \underset{\left(\left(M_{\alpha \beta}^{k}\right)_{r}\right)_{i}<0-\left(\left(M_{\alpha \beta}^{k}\right)_{s}\right)_{i}}{\operatorname{argmin}} \frac{\left(q_{\alpha}^{k}\right)_{i}}{=} \\
& -\underset{\left(\left(B^{T} B\right)^{-1} B^{T} N_{i}\right)_{i}<0}{\operatorname{argmin}} \frac{\left(\left(B^{T} B\right)^{-1}\right)_{i}}{\left(\left(B^{T} B\right)^{-1} B^{T} N_{s}\right)_{i}} \\
= & \underset{\left(d_{D}\right)_{i}<0}{\operatorname{argmin}} \frac{\left(x_{B}\right)_{i}}{-\left(d_{D}\right)_{i}},
\end{aligned}
$$

where $d_{D}=-\left(B^{T} B\right)^{-1} B^{T} N_{s}$. On the other hand, the least-squares algorithm will select $x_{r}$ to become nonbasic, where

$$
r=\underset{y_{i}<0}{\operatorname{argmax}} \frac{-y_{i}}{\left(x_{B}\right)_{i}-y_{i}}=\underset{y_{i}<0}{\operatorname{argmin}} \frac{\left(x_{B}\right)_{i}}{-y_{i}}
$$




$$
y=\left(\left(\begin{array}{c}
B^{T} \\
N_{s}^{T}
\end{array}\right)\left(\begin{array}{ll}
B & N_{s}
\end{array}\right)\right)^{-1}\left(\begin{array}{c}
B^{T} \\
N_{s}^{T}
\end{array}\right) b .
$$

From Theorem 2.24 we know that the last component of $y$ is strictly positive, and is thus not considered in the minimum-ratio test. After much algebraic manipulation, we can again rewrite the expression for this minimum-ratio test as

$$
r=\underset{\left(x_{b}-\gamma d_{D}\right)_{1}, 00}{\operatorname{argmin}} \frac{\left(x_{B}\right)_{i}}{-\left(x_{B}-\gamma d_{D}\right)_{i}}
$$

where

$$
\gamma=\frac{c}{d} ; \quad c=N_{s}^{T} u ; \quad d=N_{s}^{T} B\left(B^{T} B\right)^{-1} B^{T} N_{s}-N_{s}^{T} N_{s} .
$$

In order to demonstrate that both minimum-ratio tests must select the same index $r$, we will derive conditions that must be met in order for a different index to be chosen. This will produce a contradiction.

Assume that for some indices $j$ and $k$, we have

$$
\frac{\left(x_{B}\right)_{j}}{-\left(d_{D}\right)_{j}}>\frac{\left(x_{B}\right)_{k}}{-\left(d_{D}\right)_{k}} \quad \text { and } \quad \frac{\left(x_{B}\right)_{j}}{-\left(x_{B}-\gamma d_{D}\right)_{j}}<\frac{\left(x_{B}\right)_{k}}{-\left(x_{B}-\gamma d_{D}\right)_{k}}
$$

where

$$
\left(d_{D}\right)_{j}<0 ; \quad\left(d_{D}\right)_{k}<0 ; \quad\left(x_{B}-\gamma d_{D}\right)_{j}<0 ; \quad\left(x_{B}-\gamma d_{D}\right)_{k}<0 .
$$

This can only be true if we have both

$$
\frac{-\left(d_{D}\right)_{j}}{\left(x_{B}\right)_{j}}<\frac{-\left(d_{D}\right)_{k}}{\left(x_{B}\right)_{k}} \quad \text { and } \quad \frac{-\gamma\left(d_{D}\right)_{j}}{\left(x_{B}\right)_{j}}<\frac{-\gamma\left(d_{D}\right)_{k}}{\left(x_{B}\right)_{k}}
$$

which can only be true if $\gamma>0$ and

$$
\begin{aligned}
& \left(d_{D}\right)_{j}<0 \quad \text { and } \quad\left(d_{D}\right)_{j}>\frac{1}{\gamma}\left(x_{B}\right)_{j} \\
& \left(d_{D}\right)_{k}<0 \quad \text { and } \quad\left(d_{D}\right)_{k}>\frac{1}{\gamma}\left(x_{B}\right)_{k} .
\end{aligned}
$$

We know that the: all of these conditions cannot be satisfied simultaneously if $\gamma>0$, as we know $x_{B}>0$. Therefore, both algorithms select the same index $r$, thus choosing the same basic $x$ variable to become nonbasic.

At this point, the basic variable $x_{r}$ is dropued from the basis in both algorithms, moving index $r$ from the basic to the nonbasic index set. In the quadratic programming algorithm, the next outgoing (blocking) basic variable must be determined, as $x_{s}$ has not yet been brought into the basis. In the leastsquares algorithm, things are slightly different because the incoming variable $x_{s}$ is already basic. However, once $x_{r}$ is dropped from the basis and a new value for 
$y$ is computed by the least-squares algorithm, we are back in the same situation as described earlier. This time we have a smaller set of basic variables, and by the same argument given above, both algorithms will again select the same basic $x$ variable to leave the basis. Thus we may conclude that given the same initial index sets $\alpha$ and $\beta$, the same incoming column is selected, and the same series of variables is dropped from the basis. Once all necessary basic columns have been dropped, the quadratic programming algorithm finally brings $x_{s}$ into the basis, and both algorithms again share the same sets of basic and nonbasic indices.

Therefore we see that the only difference between the two algorithms is the fact that after $x_{s}$ has been successfully introduced into the basis (and all dropping of basic $x$ variables is commplete), the least-squares algorithm takes all basic. variables with a zero value an makes them nonbasic, while the quadratic programming algorithm does not. In this degenerate situation, the two algorithms can develop different index sets, and can thus take a different path to find a different final solution.

Notice that we have also just proved that this quadratic programming algorithm is equivalent to the NNLS algorithm in Lawson \& Hanson [12].

\section{Computational Results}

In this section, we consider the actual performance of the algorithm developed in Section 2. All computational results have been obtained using the 30 smallest linear programming test problems available from $N E T L I B$ [7]. These test problems were converted to the form of (2-1), with the addition that free variables were allowed (although nonpositive variables were not). This produced a set of equivalent problems with modified dimensions; see Table 3-1 for the original and modified dimensions. For bitmaps of the nonzero patterns of many of the original problems, see [13].

The test environment consisted of the following hardware and software:

Computer:

HP 9000/835

$32 \mathrm{M}$ main memory

$100 \mathrm{M}$ virtual mernory

Operating System: HP-UX 7.0

Language: HP FORTRAN, 64-bit real numbers

The run times reported here include only computation time, as the amount of main memory used virtually eliminated swapping, and $\mathrm{I} / \mathrm{O}$ time was negligible.

\subsection{The Least-Squares Phase I Algorithm}

This section considers the computational results of the least-squares Phase I algorithm and its variations, as developed in Section 2. See Sections 2.8 and 2.9 for pseudo code of the algorithm, and a discussion of the variations. We do not 


\begin{tabular}{|l|rr|rr|}
\hline & \multicolumn{2}{|c|}{ Original } & \multicolumn{2}{c|}{ Converted } \\
\hline Problem(Name) & Rows & Columns & Rows & Columns \\
\hline 1(AFIRO) & 28 & 32 & 28 & 51 \\
2(SC50A) & 51 & 48 & 51 & 78 \\
3(SC5OB) & 51 & 48 & 51 & 78 \\
4(ADLITTLE) & 57 & 97 & 57 & 138 \\
5(BLEND) & 75 & 83 & 75 & 114 \\
\hline 6(SHARE2B) & 97 & 79 & 97 & 162 \\
7(SC105) & 106 & 103 & 106 & 163 \\
8(STOCFOR1) & 118 & 111 & 118 & 165 \\
9(RECIPE) & 92 & 180 & 212 & 298 \\
10(SCAGR7) & 130 & 140 & 130 & 185 \\
\hline 11(BOEING2) & 167 & 143 & 244 & 382 \\
12(ISRAEL) & 175 & 142 & 175 & 316 \\
13(SHARE1B) & 118 & 225 & 118 & 253 \\
14(VTP.BASE) & 199 & 203 & 346 & 475 \\
15(SC205) & 206 & 203 & 206 & 317 \\
\hline 16(GROW7) & 141 & 301 & 421 & 581 \\
17(BEACONFD) & 174 & 262 & 174 & 295 \\
18(BRANDY) & 221 & 249 & 221 & 303 \\
19(SCSD1) & 78 & 760 & 78 & 760 \\
20(E226) & 224 & 282 & 224 & 472 \\
\hline 21(FORPLAN) & 162 & 421 & 187 & 514 \\
22(BORE3D) & 234 & 315 & 247 & 346 \\
23(AGG) & 489 & 163 & 489 & 615 \\
24(CAPRI) & 272 & 353 & 419 & 613 \\
25(SCORPION) & 389 & 358 & 389 & 466 \\
\hline 26(BANDM) & 306 & 472 & 306 & 472 \\
27(SCTAP1) & 301 & 480 & 301 & 660 \\
28(SCFXM1) & 331 & 457 & 331 & 600 \\
29(STAIR) & 357 & 467 & 445 & 620 \\
30(SCSD6) & 148 & 1350 & 148 & 1350 \\
\hline
\end{tabular}

Table 3-1: Size of Test Problems

consider the "pure" algorithm at all, as the variation allowing free variables is always more efficient when they are present. Thus we will consider the following four least-squares algorithms:

P1F: The least-squares Phase I algorithm, in which free variables have not been explicitly converted to pairs of nonnegative variables.

P1D1F: The same as PIF, with the exception that the denominator of the column selection rule is replaced by "l".

CBP1F: The same as P1F, using a crash basis as described in Section 2.9.

CBP1D1F: The same as P1D1F, using a crash basis as described in Section 2.9 . 
All of these least-squares Phase I algorithms were implemented using dense matrix methods. QR factorization was used to solve the embedded least-squares subproblems, and this factorization was updated using Givens rotations as columns were added and dropped from the basis. See for example [9] for details on using the QR factorization to solve least-squares problems, and for details on Givens rotations (also known as plane rotations).

The least-squares algorithms are compared with LSSOL, an established package. LSSOL is written in Fortran 77 using dense matrix techniques, and solves a class of linearly constrained quadratic programming problems. See [8] for a more detailed description of LSSOL. In order to compare our Phase I algorithm to LSSOL, we use the "FS" option. This causes LSSOL to find a feasible solution to the problem submitted, using its implementation of Phase $\mathbf{I}$ of the simplex method. LSSOL (FS option) will be denoted by FS in the following result tables, and by LSSOL:FS in the text.

When looking at the least-squares Phase I algorithms, we see that there is no clear-cut definition for an iteration, because the loop in which columns are dropped from the basis could be executed a large number of times between column selections. We decided to define an iteration to occur whenever a leastsquares problem is solved. This means that adding a single column to the basis is considered to be an iteration, as is dropping a column by forming a convex combination (see Step 3 of the algorithm). In addition, consider the situation in which some elements of the solution are 0 , and are consequently dropped. Although no least-squares problem is solved in this case, enough work is performed in matrix updates, etc., that this is also considered to be an iteration.

Table 3-2 displays the iteration counts of the four least-squares Phase I algorithms and LSSOL:FS. Note that the iteration counts for some of the problems are the same for both the non-crash-basis and crash-basis versions of the leastsquares Phase I algorithm (e.g. STOCFOR1). This is due to the fact that not all problems contain columns of the form we look for when building the crash basis. Also notice that some of the iteration counts are 0 . This indicates the situation when the initial crash basis provides a feasible solution, and no iterations are necessary.

We see from Table 3-2 that LSSOL:FS takes a number of iterations comparable to that taken by the least-squares Phase 1 algorithms, with the total lying between P1D1F and CBP1F. As stated earlier, the notion of an iteration in the least-squares Phase I algorithms is somewhat nonstandard. For this reason, actual run times are probably a more accurate measure of the least--squares algorithms' performance than their iteration counts.

Table 3-3 displays the run times of the four least-squares Phase I algorithms and LSSOL:FS, reported in CPU-seconds. Note that a number of runtimes are reported as " 0 ". This indicates that the CPU-times recorded for these problems round down to less than one CPU-second. We see from Table 3-3 that the least-squares algorithms run in substantially less time than does LSSOL:FS; 


\begin{tabular}{|c|c|c|c|c|c|}
\hline Problem(Nane) & $\mathrm{P} 1 \mathrm{~F}$ & P1D1F & CBP1F & CBP1D1F & $\overline{F S}$ \\
\hline 1 (AFIRO) & 7 & 7 & 0 & 0 & 8 \\
\hline $2(\mathrm{SC} 50 \mathrm{~A})$ & 20 & 20 & 0 & 0 & 3 \\
\hline $3(\mathrm{SC} 50 \mathrm{~B})$ & 5 & 5 & 0 & 0 & 0 \\
\hline 4 (ADLITTLE) & 56 & 56 & 27 & 27 & 70 \\
\hline 5(BLEND) & 8 & 8 & 10 & 0 & 49 \\
\hline 6(SHARE2B) & 248 & 248 & 229 & 217 & 74 \\
\hline $7(\mathrm{SC} 105)$ & 34 & 34 & 0 & 0 & 2 \\
\hline 8(STOCFOR1) & 98 & 98 & 98 & 98 & 44 \\
\hline 9(RECIPE) & 106 & 106 & $3 T_{1}$ & 37 & 46 \\
\hline 10(SCAGR7) & 169 & 169 & 145 & 145 & 22 \\
\hline 11(BOEING2) & 206 & 204 & 163 & 161 & 181 \\
\hline 12(ISRAEL) & 171 & 171 & 8 & 8 & 338 \\
\hline 13(SHARE1B) & 189 & 189 & i76 & 176 & 150 \\
\hline 14(VTPBASE) & 770 & 561 & 488 & 507 & 1059 \\
\hline $15(\mathrm{SC} 205)$ & 61 & 61 & $\mathbf{0}$ & 0 & 2 \\
\hline 16(GROW 7) & 280 & 280 & $\overline{0}$ & 0 & 203 \\
\hline 17(BEACONFD) & 122 & 122 & 89 & 89 & + 52 \\
\hline 18(BRANDY) & 365 & 365 & 340 & 340 & 300 \\
\hline 19(SCSD1) & 10 & 8 & 10 & 8 & 16 \\
\hline 20(E226) & 203 & 203 & 154 & 154 & 209 \\
\hline 21 (FORPLAN) & 378 & 378 & 347 & 347 & 438 \\
\hline 22(BORE3D) & 175 & 175 & 164 & 164 & 126 \\
\hline 23(AGG) & 543 & 5.39 & 134 & 136 & 149 \\
\hline 24(CAPRI) & 586 & 590 & 440 & 444 & 451 \\
\hline $25(\mathrm{SCORPION})$ & 321 & 321 & 293 & 293 & 27 \\
\hline $26(\mathrm{BANDM})$ & 484 & 485 & 419 & 419 & 369 \\
\hline $27($ SCTAP 1$)$ & 440 & 411 & 322 & 349 & 430 \\
\hline $28($ SCFXM1) & 350 & 343 & 300 & 293 & 271 \\
\hline 29 (STAIR) & 452 & 452 & 464 & 464 & 173 \\
\hline 30(SCSD6) & 207 & 68 & 207 & 68 & 61 \\
\hline TOTAL & 7064 & 6677 & 5054 & 4944 & 5323 \\
\hline
\end{tabular}

Table 3-2: Least-Squares Phase I Algorithm Iteration Counts

the LSSOL:FS total time is almost 3.5 times longer than the total of the best least-squares Phase I algorithm (CBP1D1F). In fact, LSSOL:FS solved only one problem (SCAGR7) faster than did CBP1D1F.

Looking at the least-squares algorithms more closely, the "denominator-of-1" versions (P1D1F and CBP1D1F) are quite similar to their "full denominator" counterparts (P1F and CBP1F). However, the crash basis versions (CBP1F and (CBPID1F) are noticeably faster than the "from scratch" versions (PIF and P1D1F).

So far, we have discussed iteration count and run times. We will now consider in some sense the path taken by the least-squares Phase I algorithms. In particular, we will consider the norm of the residual (error vector) as a function of iteration count. For the least-squares Phase I algorithms, this is $\|u\|$, where $u$ is as defined in the pseudocode algorithm found in Section 2.8 


\begin{tabular}{|l|rrrr|r|}
\hline Problem(Name) & P1F & P1D1F & CBP1F & CBP1D1F & FS \\
\hline 1(AFIRO) & 0 & 0 & 0 & 0 & 1 \\
2(SC50A) & 0 & 0 & 0 & 0 & 1 \\
3(SC50B) & 0 & 0 & 0 & 0 & 1 \\
4(ADLITLE) & 2 & 2 & 1 & 1 & 5 \\
5(BLEND) & 0 & 1 & 0 & 0 & 6 \\
\hline 6(SHARE2B) & 12 & 12 & 11 & 11 & 15 \\
7(SC105) & 2 & 2 & 1 & 1 & 5 \\
8(STOCFOR1) & 7 & 7 & 7 & 7 & 16 \\
9(RECIPE) & 21 & 21 & 10 & 10 & 61 \\
10(SCAGR7) & 15 & 15 & 15 & 15 & 14 \\
\hline 11(BOEING2) & 61 & 59 & 48 & 47 & 209 \\
12(ISRAEL) & 32 & 30 & 5 & 4 & 227 \\
13(SHARE1B) & 18 & 17 & 17 & 18 & 51 \\
14(VTP.BASE) & 416 & 311 & 271 & 277 & 2026 \\
15(SC205) & 11 & 11 & 2 & 2 & 34 \\
\hline 16(GROW7) & 207 & 209 & 11 & 11 & 801 \\
17(BEACONFD) & 20 & 21 & 18 & 17 & 42 \\
18(BRANDY) & 79 & 78 & 80 & 75 & 208 \\
19(SCSD1) & 3 & 3 & 4 & 3 & 7 \\
20(E226) & 63 & 61 & 51 & 51 & 196 \\
\hline 21(FORPLAN) & 98 & 95 & 90 & 90 & 398 \\
22(BORE3D) & 48 & 47 & 47 & 40 & 153 \\
23(AGG) & 676 & 686 & 182 & 181 & 889 \\
24(CAPRI) & 545 & 549 & 472 & 478 & 1438 \\
25(SCORPION) & 182 & 182 & 172 & 173 & 277 \\
\hline 26(BANDM) & 244 & 242 & 224 & 223 & 571 \\
27(SCTAP1) & 274 & 263 & 221 & 229 & 695 \\
28(SCFXM1) & 223 & 222 & 202 & 199 & 530 \\
29(STAIR) & 545 & 544 & 586 & 589 & 816 \\
30(SCSD6) & 130 & 41 & 128 & 41 & 45 \\
\hline TOTAL & 3934 & 3731 & 2876 & 2799 & 9738 \\
\hline
\end{tabular}

Table 3-3: Least-Squares Phase I Algorithm Run Times

Figure 3-1 illustrates the typical behavior of $\|u\|$ in P1F. We chose to display the behavior of only one of the four least-squares Phase I algorithms because in this aspect, they were virtually identical. Notice that P1F finds a solution "close" to feasibility very quickly, spending the vast majority of its iterations reducing $\|u\|$ from 0.1 to 0 .

The last comparison we will make between the least-squares Phase I algorithms and LSSOL:FS will be based on the accuracy of the final solution found. When each algorithm found an $\hat{x}$ that it claimed to be a feasible solution (or as close as possible to such a solution), the error quantity $\|b-A \dot{x}\|$ was formed and reported. These errors in the final solution are displayed in Table 3-4.

In most problems, there is very little difference in accuracy between the leastsquares Phase I algorithrns and LSSOL:FS. However, in the case of FORPLAN, the error of the least-squares algorithms is quite large relative to the error of 


\section{CAPRI}

llull

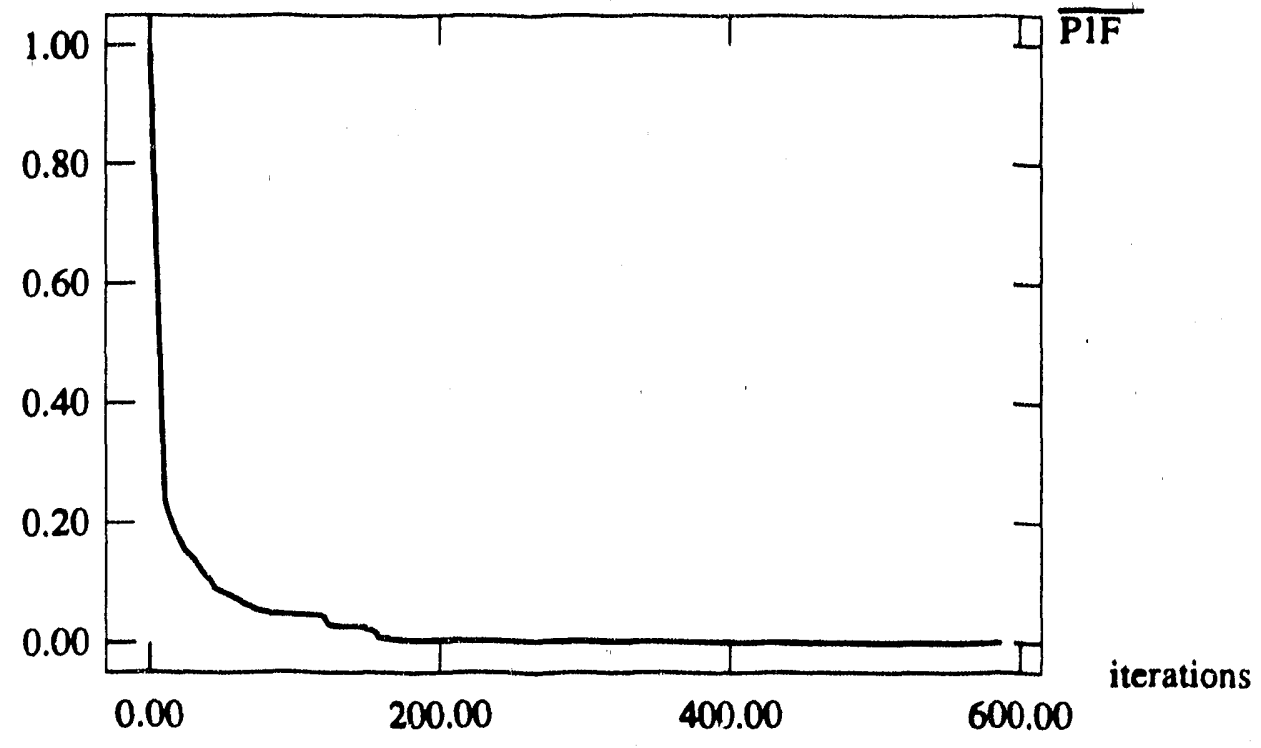

Figure 3-1: Residual Behavior of P1F on CAPRI

LSSOL:FS. This is not surprising, as the error in the computed solution $\hat{x}$ in the least-squares algorithms depends on the square of the condition number of the matrix of currently basic columns (except when $B$ is square). (See, for example [3].) This inaccuracy can occasionally cause least-squares Phase I algorithms to report infeasibility incortectly.

It may be possible to handle this lack of accuracy by adding iterative refinement to the end of the least-squares Phase I algorithms. That is, we tuke the (previously final) solution $\hat{x}$ and let $x^{0}=\hat{x}$. Let $B$ be the matrix of currently basic columns. We then perform some (small) number of iterations

$$
\begin{aligned}
u^{i} & =b-B x^{i} \\
B \delta x^{i} & =u^{i} \\
x^{i+1} & =x^{i}+\delta x^{i} .
\end{aligned}
$$

In practice, we have the $\mathrm{QR}$ factorization of $B$ as opposed to $B$, and $B$ is generally rectangular, so we would actually perform iterative refinement as follows:

$$
\begin{aligned}
u^{i} & =b-Q\left(\begin{array}{c}
R \\
0
\end{array}\right) x^{i} \\
\left(\begin{array}{c}
R \\
0
\end{array}\right) \delta x^{i} & =Q^{T} u^{i}
\end{aligned}
$$




\begin{tabular}{|c|c|c|c|c|c|}
\hline Problem(Naine) & P1F & P1D1F & CBP1F & CBP1D1F & $\overline{F S}$ \\
\hline 1 (AFIRO) & 0 & 0 & 0 & 0 & $5 e-11$ \\
\hline $2(\mathrm{SC} 50 \mathrm{~A})$ & $7 e-14$ & $7 e-14$ & 0 & 0 & $5 e-13$ \\
\hline $3(\mathrm{SC} 50 \mathrm{~B})$ & 0 & 0 & 0 & 0 & $4 e-14$ \\
\hline $\mathbf{4}(\mathrm{ADLITTLE})$ & $3 e-13$ & $3 e-13$ & $2 e-13$ & $2 e-13$ & $7 e-12$ \\
\hline 5(BLEND) & 0 & 0 & 0 & 0 & $9 e-12$ \\
\hline 6(SHARE2B) & $2 e-12$ & $1 e-12$ & $1 e-12$ & $2 \mathrm{e}-12$ & $6 \mathrm{e}-11$ \\
\hline $7(\mathrm{SC105})$ & $4 e-13$ & $4 e-13$ & 0 & 0 & $2 e-12$ \\
\hline 8(STOCFOR1) & $4 e-14$ & $4 e-14$ & $4 e-14$ & $4 \mathrm{e}-14$ & $2 e-10$ \\
\hline 9(RECIPE) & $1 e-14$ & $1 e-14$ & $1 e-14$ & $1 \mathrm{e}-14$ & $3 e-13$ \\
\hline 10 (SCAGR7) & $5 e-12$ & $8 e-12$ & $6 e-12$ & $7 e-12$ & $2 \mathrm{e}-10$ \\
\hline j1(BOEING2) & $1 \mathrm{e}-11$ & $3 e-11$ & $4 e-11$ & $9 e-12$ & $1 \mathrm{e}-10$ \\
\hline 12 (ISRAEL) & $3 e-10$ & $5 e-10$ & $1 e-10$ & $1 e-10$ & $5 e-09$ \\
\hline 13(SHARE1B) & $3 e-10$ & $3 e-10$ & $2 e-10$ & $2 e-10$ & $9 e-09$ \\
\hline 14(VTP.BASE) & $7 e-10$ & $8 e-10$ & $1 e-09$ & $2 e-09$ & $9 e-09$ \\
\hline $15(\mathrm{SC} 205)$ & $1 \mathrm{e}-12$ & $1 e-12$ & 0 & 0 & $3 e-11$ \\
\hline $16(\mathrm{GROW} 7)$ & 0 & $\overline{0}$ & 0 & 0 & $1 \mathrm{e}-08$ \\
\hline 17(BEACONFD) & $6 e-11$ & $6 e-11$ & $6 e-11$ & $6 e-11$ & $6 e-10$ \\
\hline 18(BRANDY) & $3 e-12$ & $3 e-12$ & $1 e-10$ & $1 e-10$ & $5 e-11$ \\
\hline 19(SCSD1) & $3 e-16$ & $2 e-16$ & $3 e-16$ & $2 e-16$ & $2 e-15$ \\
\hline 20(E226) & $5 e-13$ & $2 e-13$ & $7 e-14$ & $7 e-14$ & $2 \mathrm{e}-11$ \\
\hline 21(FORPLAN) & $8 e-01$ & $8 e-01$ & $8 \mathrm{e}-01$ & $8 \mathrm{e}-01$ & $9 \mathrm{e}-10$ \\
\hline 22(BORE3D) & $7 e-12$ & $7 e-12$ & $7 e-12$ & $7 e-12$ & $3 e-09$ \\
\hline $23(\mathrm{AGG})$ & $1 e-07$ & $1 e-07$ & $1 e-09$ & $1 e-09$ & $1 e-08$ \\
\hline 24(CAPRI) & $6 e-11$ & $6 e-11$ & $1 e-10$ & $1 e-10$ & $3 e-09$ \\
\hline 25 (SCORPION) & $2 e-15$ & $2 e-15$ & $2 \mathrm{e}-15$ & $2 \mathrm{e}-15$ & $2 \mathrm{e}-14$ \\
\hline $26(\mathrm{BANDM})$ & $1 \mathrm{e}-11$ & $6 e-11$ & $2 e-11$ & $1 e-11$ & $4 e-11$ \\
\hline $27($ SCTAP1) & $6 e-12$ & $3 e-12$ & $4 e-12$ & $7 e-12$ & $5 e-12$ \\
\hline $28(\mathrm{SCFXM1})$ & $3 e-12$ & $2 e-11$ & $2 e-11$ & $2 e-11$ & $9 e-10$ \\
\hline 29(STAIR) & $7 e-13$ & $6 e-13$ & $2 e_{-} 12$ & $2 e-12$ & $3 e-09$ \\
\hline 30(SCSD6) & $1 e-15$ & $2 \mathrm{e}-15$ & $1 \mathrm{e}-15$ & $2 e-15$ & $2 \mathrm{e}-14$ \\
\hline
\end{tabular}

Table 3-4: Least-Squares Phase I Algorithm Accuracy

$$
x^{i+1}=x^{i}+\delta x^{i} .
$$

As long as $Q$ is stored, this would not be too much work, especially if only a few iterations were necessary. Note that this scheme for iterative refinement depends on $B x=b$ being nearly feasible. This may not be the case if we have accumulated a significant amount of error. See [2] for more details, as well as for the description of a method of iterative refinement that is not dependent on the feasibility of $B x=b$.

To surnmarize, the four least-squares Phase I algorithms have very favorable run times relative to the simplex method as implernented by LSSOL, with the "crash basis" versions performing the best. They also find a solution "close" to feasibility very quickly. However, due to the least-squares subproblems enbedded in the four Phase I algorithms, accuracy can be a problem on larger, more ill-conditioned problems. It is possible that iterative refinement of the final 


\begin{tabular}{|c|c|c|c|c|c|}
\hline Problem(Name) & $P: F$ & P1DIF & CBPIF & CBP1DIF & LS \\
\hline 1 (AFIRO) & 7 & 7 & 0 & 0 & 22 \\
\hline $2(\mathrm{SC} 50 \mathrm{~A})$ & 20 & 20 & 0 & 0 & 38 \\
\hline $3(\mathrm{SC} 50 \mathrm{OB})$ & 5 & 5 & 0 & 0 & 26 \\
\hline $4($ ADLITTLE) & 56 & 56 & 27 & 27 & 148 \\
\hline 5(BLEND) & 8 & 8 & 0 & 0 & 17 \\
\hline 6(SHARE2B) & 248 & 248 & 229 & 217 & 243 \\
\hline $7(\mathrm{SC} 105)$ & 34 & 34 & 0 & 0 & 88 \\
\hline $8($ STOCFOR1) & 98 & 98 & 98 & 98 & 149 \\
\hline 9 (RECIPE) & 106 & 106 & 37 & 37 & 204 \\
\hline 10 (SCAGR7) & 169 & 169 & 145 & 145 & 251 \\
\hline 11 (BOEING2) & 206 & 204 & 163 & 161 & 925 \\
\hline $12($ ISR & 171 & 171 & 8 & 8 & 836 \\
\hline 13(SHAKE1B) & 189 & 189 & 176 & 176 & 409 \\
\hline 14(VTP.BASE) & 770 & 561 & 488 & 507 & 2499 \\
\hline $15(\mathrm{SC} 205)$ & 61 & 61 & 0 & 0 & 218 \\
\hline 16(GROW7) & 280 & 280 & 0 & 0 & 620 \\
\hline 17 (BEACONFD) & 122 & 122 & 89 & 89 & 214 \\
\hline 18(BRANDY) & 365 & 365 & 340 & 340 & 598 \\
\hline $19(\mathrm{SCSD} 1)$ & 10 & 8 & 10 & 8 & 35 \\
\hline 20(E226) & 203 & 203 & 154 & 154 & 399 \\
\hline $21(\mathrm{FORPLAN})$ & $\overline{378}$ & 378 & 347 & 347 & 436 \\
\hline 22(BORE3D) & 175 & 175 & 164 & 164 & 385 \\
\hline $23(A G G)$ & 543 & 539 & 134 & 136 & 608 \\
\hline $24(C A P R I)$ & 586 & 590 & 440 & 444 & 778 \\
\hline 25 (SLORPION) & 321 & 321 & 293 & 293 & 320 \\
\hline 26(BANDM) & 484 & 485 & 419 & 419 & 874 \\
\hline 27 (SC'TAP1) & 440 & 411 & 322 & 349 & 1210 \\
\hline $28(S C F X M 1)$ & 350 & 343 & 300 & 293 & 520 \\
\hline $29(\mathrm{STAIR})$ & 452 & 452 & 464 & 464 & 679 \\
\hline $30($ SCSD 6$)$ & 207 & 68 & 207 & 68 & 178 \\
\hline TOTAL & 7064 & 6677 & 5054 & 4944 & 13927 \\
\hline
\end{tabular}

Table 3-5: Phase I Algorithm and LSSOL:LS Iteration Counts

solution could alleviate such difficulties, but this variation was not implemented.

\subsection{A Constrained Least Squares Problem}

So far we have considered computation results on problem (2-1). However, as we noted in Section 2.10, our least-squares Phase I algorithm actually solves (215), a constrained least-squares probl.m. Thus we decided to compare our Phase I algorithm with that of LSSOL when applied to (2-15) (by using the "LS" option). LSSOL (LS option) will be denoted by LS in the following resuli tables, and by LSSOL:LS in the text.

Table 3-5 displays the iteration counts of the least-squares Phase I algorithms considered in Section 3.1 and LSSOL:LS, and Table 3-6 displays the run times. Again, the least-squares Phase I algorithms do well, with the best, (BPIDIF. 


\begin{tabular}{|c|c|c|c|c|c|}
\hline Problem(Name) & $\mathrm{P} 1 \mathrm{~F}$ & P1D1F & CBP1F & CBP1D1F & $\overline{\mathrm{LS}}$ \\
\hline 1(AFIRO) & 0 & 0 & 0 & 0 & 1 \\
\hline $2(\mathrm{SC} 50 \mathrm{~A})$ & $\mathbf{0}$ & 0 & 0 & 0 & 1 \\
\hline $3(\mathrm{SC} 50 \mathrm{~B})$ & 0 & $\mathbf{0}$ & 0 & o & 1 \\
\hline 4(ADLITTLE) & 2 & 2 & 1 & 1 & 5 \\
\hline 5(BLEND) & 0 & 1 & 0 & $\mathbf{0}$ & 2 \\
\hline 6(SHARE2B) & 12 & 12 & 11 & 11 & 11 \\
\hline 7 (SC105) & 2 & 2 & 1 & 1 & 7 \\
\hline 8(STOCFOR1) & 7 & 7 & 7 & 7 & 10 \\
\hline 9(RECIPE) & 21 & 21 & 10 & 10 & 49 \\
\hline 10(SCAGR7) & 15 & 15 & 15 & 15 & 19 \\
\hline 11(BOEING2) & 61 & $\overline{59}$ & 48 & 47 & 214 \\
\hline 12(ISRAEL) & 32 & 30 & 5 & 4 & 109 \\
\hline 13(SHAREIB) & 18 & 17 & 17 & 18 & 31 \\
\hline 14(VTP.BASE) & 416 & 311 & 271 & 277 & 812 \\
\hline $15(\mathrm{SC} 205)$ & 11 & 11 & 2 & 2 & 51 \\
\hline 16(GROW7) & 207 & 209 & 11 & 11 & 409 \\
\hline 17(BEACONFD) & 20 & 21 & 18 & 17 & 55 \\
\hline 18(BRANDY) & 79 & 78 & 80 & 75 & 109 \\
\hline 19 (SCSD 1$)$ & 3 & 3 & 4 & 3 & 47 \\
\hline $20(\mathrm{E} 226)$ & 63 & 61 & 51 & 51 & 151 \\
\hline 21(FORPLAN) & 98 & 95 & 90 & 90 & 174 \\
\hline 22(BORE3D) & 48 & 47 & 47 & 46 & 120 \\
\hline $23(\mathrm{AGG})$ & 676 & 686 & 182 & 181 & 608 \\
\hline 24(CAPRI) & 545 & 549 & 472 & A7o & 529 \\
\hline 25(SCORPION) & 182 & 182 & 172 & 173 & 217 \\
\hline 26(BANDM) & 244 & 242 & 224 & 223 & 409 \\
\hline 27(SCTAP1) & 274 & 263 & 221 & 229 & 711 \\
\hline 28(SCFXM1) & 223 & 222 & 202 & 199 & 400 \\
\hline 29(STA IR) & 545 & 544 & 586 & 589 & 573 \\
\hline $30($ SCSD6) & 130 & 41 & 128 & 41 & 342 \\
\hline TOTAL & 3934 & 3731 & 2876 & 2799 & 6177 \\
\hline
\end{tabular}

Table 3-6: Phase I Algorithm and LSSOL:LS Run Times

running in $45 \%$ of the total time taken by LSSOL:LS. Apparently, our leastsquares Phase I algorithms are also efficient in solving this particular kind of constrained least-squares problem.

\section{Summary and Conclusions}

Section 2 developed a Phase I algorithm using least-squares subproblems. Subproblems are solved to provide both an approximation to the right-hand side that is as close as possible in the 2-norm (given the current basic columns), and to select the next incoming column. The incoming column-selection rule is myopic, selecting that column whose nonnegative combination with the current approximaiion brings us closest to the right-hand side. This Phase I algorithm 
has the property of strict improvement at each iteration, even in the presence of degeneracy (in the simplex method sense). Finally, equivalences between the least-squares Phase I algorithm and other algorithms were discussed.

Four variations of this least-squares Phase I algorithm were developed, and the computational results were excellent. The best least-squares Phase I algorithm ran almost 3.5 times faster than LSSOL's implementation of Phase I of the simplex method. In addition, the same algorithm ran 2.2 times faster than LSSOL's constrained LS solver, when applied to problems of the form (2-15).

\section{Future Work}

We have demonstrated the initial proof-of-concept of the Phase I algorithn developed in Section 2. In ordey" for this algorithm to be commercially competitive, it must be fine-tuned, just as the simplex method has been since its discovery. In particular, the problem of inaccuracy on unstable problems must be addressed, perhaps with the inclusion of iterative refinement of the solutions to the embedded least-squares subproblems. In addition, these algorithms should be implemented using sparse matrix methods to see how they compare to sparse matrix implementations of the simplex method.

\section{References}

(1) E.M.L. Beale (1955), "Cycling in the Dual Simplex Algorithm", Naval Res. Logist. Quart., 2(4), 1955, pp.269-276.

[2] A. Björck (1967/68), "Iterative Refinement of Linear Least Squares Solutions", BIT 7, pp. 257-278 and BIT 8, pp. 8-30.

[3] A. Björck (1987), "Least Square Methods", Working Paper, Department of Mathematics, Linköping University, S-581 8.3 Linköping, Sweden, 1987.

[4] R.W. Cottle, J.S.Pang \& R.E. Stone, The Linear Ciomplementarity Problem, Academic Press, forthcoming.

[5] G.B. Dantzig (1963), Linear Programming and Extensions, Princeton University Press, Princeton, New Jersey.

[6] D. Gale (1960), Theory of Linear Economic Models, McGiaw-Hill, New York.

[7] D.M. Gay (1985). "Electronic Mail Distribution of Linear Programming 'Test Problems", Mathematical Programming Socicty COAL Newsletter 13. pp. 10-12. 
[8] P.E. Gill, S.J. Hammarling, W. Murray, M.A. Saunders \& M.H. Wright (1986), "User's Guide for LSSOL", Technical Report SOL 86-1, Department of Operations Research, Stanford University.

[9] P.E. Gill, W. Murray \& M.H. Wright (1981), Practical Optimization, Academic Press, San Francisco.

[10] Goldfarb \& Idnani(1983), "A Numerically Stable Dual Method for Solving Strictly Convex Quadratic Programs", Math. Prog. 27, pp.1-33.

[11] A.J. Hoffman (1953), "Cycling in the Simplex Algorithm", National Bureau of standards, Report No. 2974, December 16, 1953, 7 pp.

[12] C.L. Lawson \& R.J. Hanson (1974), Solving Least-Squares Problems, Prentice-Hall, Englewood Cliffs, New Jersey.

[13] I.J. Lustig (1987), "An Analysis of an Available Set of Linear Programming Test Problems", Technical Report SOL 87-11, August 1987, Department of Operations Research, Stanford University.

[14] C. Van de Panne \& A. Whinston (1969), "The Symmetric Foundation of the Simplex Method for Quadratic Programming", Econometrica, Vol. 37, pp.507-527. 


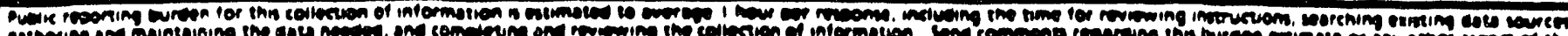

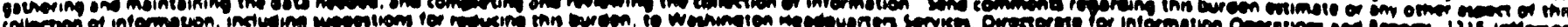

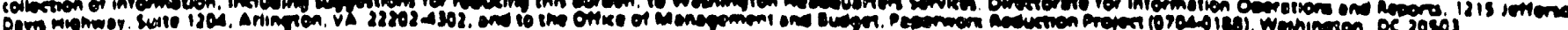

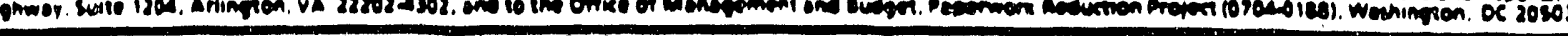

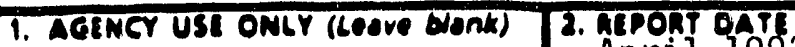

Apri1 1992

3. REPORT TYPL AND DATES COVInED

\title{
4. TITLI AND SUUTITLI
}

Technical Report

A Strictly Improving Phase I Algorithm Using Least-Squares Subproblems

5. PUMDING NUMIERS

\section{AUTHOR(S)}

NO00 14-39-J-1659

S. A. Leichner, G. B. Dantzig and J. W. Davis

DE-FG03-92ER25116

\author{
7. PERFORMING ORGANIZATION NAMI(S) AND ADORESS(ES) \\ Department of Operations Research - SOL \\ Stanford University \\ Stanford, CA 94305-4022
}

Q. PERFOAMING ORGANIZATION

REPOAT MUMBER

$1111 \mathrm{MA}$

\section{SPONSORING MONITORING AGENCY MAME(S) ANO ADDRESS(ES)}

Office of Naval Research - Department of the Navy 000 N. Quincy Street

Arlington, VA 22217

SOL $92-1$

Office of Energy Research

U.S. Department of Energy

Washington, DC 20585

120. OISTRIBUTION AVAILABILITY STATEMEN

12b. DISTRIDUTION CODE

UNL IMI TED

O. SPONSORING/MONITORING AGENCY REPORT MUMBER

13. ABSTRACT (Moximum 200 wores)

Although the simplex method's performance in solving linear programming problems is usually quite good, it does not guarantee strict improvement at each iteration on degenerate problems. Instead of trying to recognize and avoid degenerate steps in the simplex method (as some variants do), we have developed a new Phase 1 algorithm that is completely impervious to degeneracy, with a strict improvement attained at each iteration. It is also noted that the new Phase I Algorithm is closely related to a number of existing algorithms.

When tested on the 30 smallest NETLIB linear prograggming test problems, the computational results for the new Phase I algorithm were almost 3.5 times faster than the simplex method; on some problems, it was over 10 times faster.

\begin{tabular}{|c|c|c|c|}
\hline & \multirow{2}{*}{$\begin{array}{l}\text { 15. NUMBER OF PAGES } \\
4.3 \text { pages } \\
\text { 16. PRICE CODE }\end{array}$} \\
\hline \multicolumn{3}{|l|}{$\begin{array}{l}\text { Phase I. } \\
\text { Phaning; }\end{array}$} & \\
\hline
\end{tabular}



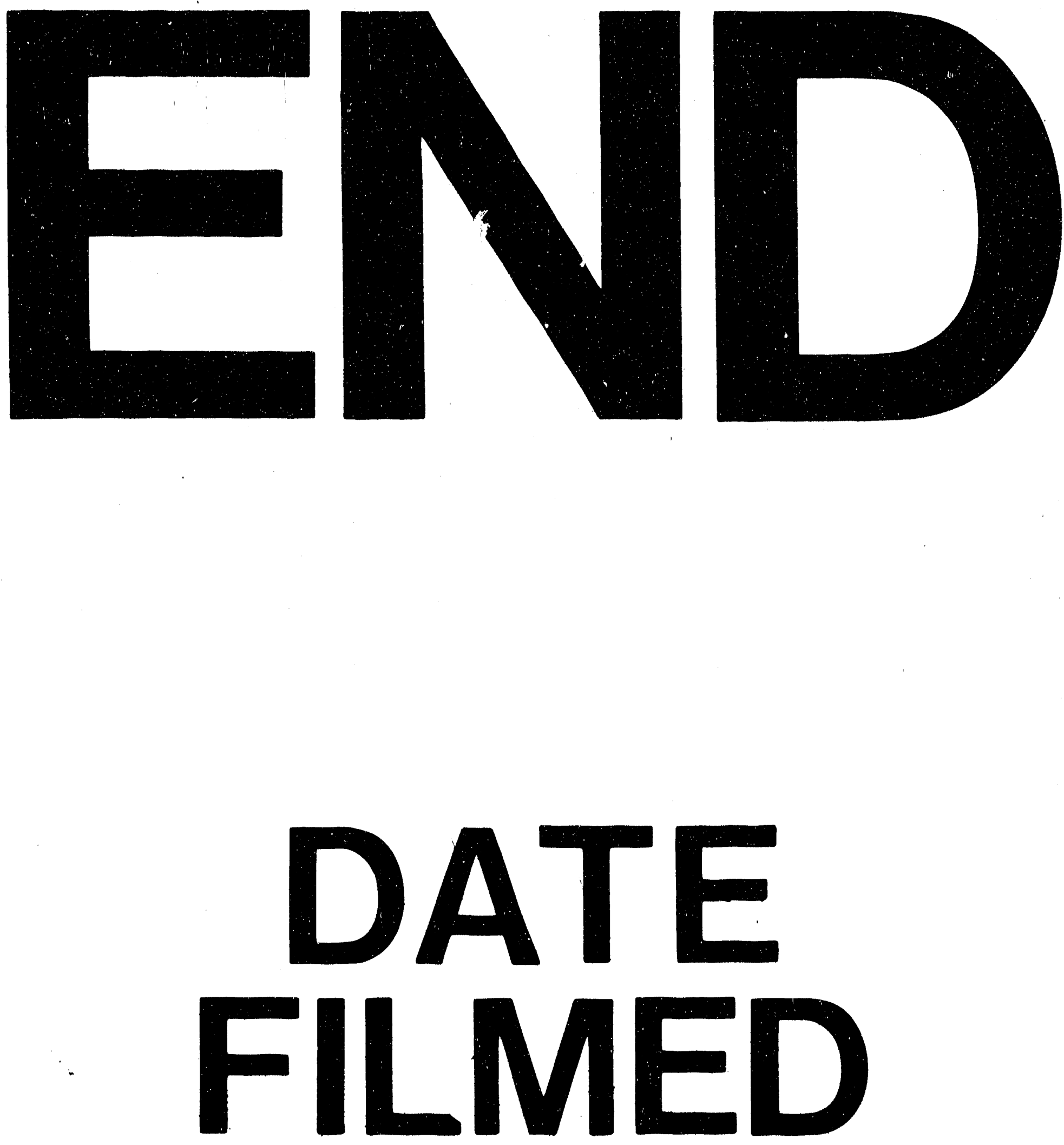

t

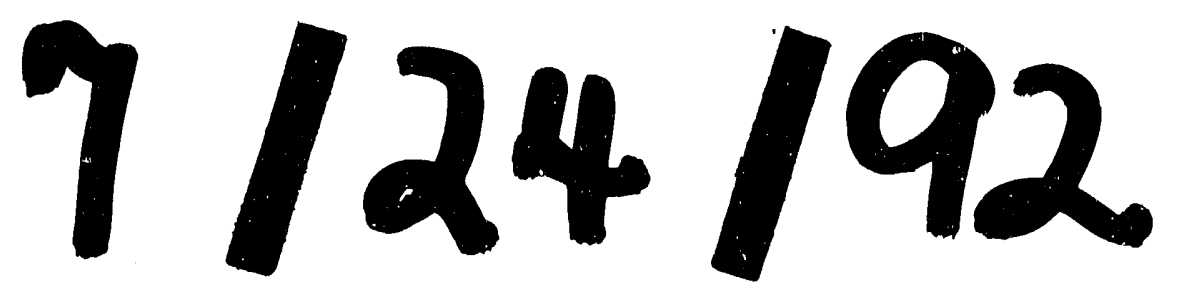


